\title{
Bubbles and cycles in the Solow-Swan model
}

\author{
Gerhard Sorger ${ }^{1}[$ (])
}

Received: 22 February 2018 / Accepted: 17 September 2018 / Published online: 25 September 2018 (c) The Author(s) 2018

\begin{abstract}
We consider a neoclassical one-sector economy in which-in addition to physical capital — there exists a second asset. This asset is unproductive, cannot be consumed, and does not pay dividends. A no-arbitrage condition is imposed so that the two assets are equivalent stores of value. Finally, we assume that consumption (respectively, investment) is a fixed fraction of the sum of aggregate factor income (GDP) and capital gains. In this modified Solow-Swan model, we characterize the conditions under which bubbles can exist, i.e., under which the useless asset can have a positive price. We find that these conditions do not imply that the original Solow-Swan equilibrium is dynamically inefficient, and we demonstrate that asset price bubbles can lead to non-monotonic and even periodic capital accumulation paths.
\end{abstract}

Keywords Solow-Swan model - Asset price bubbles · Dynamic inefficiency · Non-monotonic dynamics

JEL Classification O41 · G10 $\cdot$ E32

\section{Introduction}

The purpose of the present paper is to analyze under which conditions an intrinsically useless asset can have a positive price in a neoclassical growth model à la Solow (1956) and Swan (1956). In other words, we replicate Tirole's pathbreaking study of asset price bubbles in the overlapping generations model [see Tirole (1985)] in the simpler framework of the Solow-Swan model. Such an analysis would hardly be of any interest if it led to similar results as those derived by Tirole (1985). But this is not the case. First of all, we find that the equivalence between the existence of asset price bubbles and

The author thanks two anonymous referees and participants at the 14th Viennese Conference on Optimal Control and Dynamic Games for their constructive remarks.

\footnotetext{
$凶$ Gerhard Sorger

gerhard.sorger@univie.ac.at

1 Department of Economics, University of Vienna, Vienna, Austria
} 
the dynamic inefficiency of the standard (bubbleless) equilibrium, which is one of the central results in Tirole (1985), does not hold in our setting: bubbles can exist even if the bubbleless equilibrium is dynamically efficient. Second, we demonstrate that asset price bubbles can generate non-monotonic and even periodic capital accumulation paths, which is not the case in the overlapping generations economy studied by Tirole (1985).

We use the standard framework of a neoclassical one-sector growth model: a single output good is produced from capital and labor and it can be used for consumption and investment. We augment this setting by introducing a second asset which is intrinsically useless (it cannot be used in production, it cannot be consumed, and it does not pay any dividend). In order to ensure that the two assets are equivalent stores of value, we impose a no-arbitrage condition. Analogously to the Solow-Swan model we suppose that the amount that is consumed or invested, respectively, is a fixed fraction of aggregate income. The crucial assumption of the present paper concerns the definition of aggregate income: we assume that it consists of all factor income (i.e., GDP) plus capital gains. It follows that, whenever the price of the useless asset increases or decreases, this triggers corresponding movements of consumption and investment. It is this feedback mechanism, which opens the door for a rich set of bubbly equilibria including ones that display damped oscillations or periodic cycles. We believe that this aspect of the model, namely that capital gains may directly increase investment, is not an awkward feature of the (non-microfounded) Solow-Swan model but a rather realistic one. Moreover, the possibility of non-monotonic bubbly equilibria is in accordance with highly volatile asset price bubbles such as those observed on the markets for bitcoin or other crypto-currencies.

It has to be emphasized that the feedback loop mentioned in the previous paragraph does not exist in Tirole (1985), which is based on a two-period overlapping generations economy à la Diamond (1965). As a matter of fact, in that model the saving/investment decisions are made by the households in their first period of life, that is, at a time when their income consists only of wages. This implies that movements of the price of the useless asset do not directly affect aggregate savings (investment), but only indirectly as a general equilibrium effect.

Let us now describe our findings in more detail. We start our analysis by assuming that aggregate consumption is a fixed fraction of aggregate income. For positive population growth we find that the useless asset can have a positive price if and only if the equilibrium of the original Solow-Swan model is dynamically inefficient. In this case we therefore get the same result as Tirole (1985). If the population growth rate is negative, however, then asset price bubbles can also exist when the standard Solow-Swan equilibrium is dynamically efficient. Moreover, in this case the bubbly equilibria can display damped oscillations or even truly periodic fluctuations. We also consider a version of the model in which aggregate investment rather than aggregate consumption is a fixed fraction of GDP plus capital gains. In this situation asset price bubbles and cyclical fluctuations can occur even if the population growth rate is positive and the standard Solow-Swan equilibrium is dynamically efficient. These results demonstrate that the relation between dynamic inefficiency of capital accumulation paths and the existence of bubbles is much more subtle in our modified Solow-Swan model than in the overlapping generations framework from Tirole (1985). They also 
show that those feedback effects, which are the source of the asset price bubbles, may also be responsible for excess volatility of asset prices.

Asset price bubbles have occurred frequently in the past [see, e.g., Aliber and Kindleberger (2015) or Scherbina and Schlusche (2014, chapter 2)] and their analysis has become a hot topic of economic research. The bulk of the pertinent literature uses versions of the overlapping generations model of Samuelson (1958) and Diamond (1965) (see Tirole (1985), Weil (1987), Caballero et al. (2006), Martin and Ventura (2012, 2016), and Farhi and Tirole (2012), to name just a few). It is considerably more difficult to establish the existence of bubbly equilibria in microfounded models with infinitely-lived consumers; see Kocherlakota (1992, 2009) and Miao (2014) as well as references therein. In particular, in those models asset price bubbles can only occur in the presence of financial frictions such as collateral constraints. This is the case because without any financial frictions, bubbles would have to grow at the real interest rate and this is inconsistent with the necessary transversality condition for the consumers' optimization problem. To the best of our knowledge, asset price bubbles have not been studied in the Solow-Swan model. This is probably the case because the Solow-Swan model is not micro-founded and bubbles are thought to be episodes in which assets are voluntarily traded by rational agents despite the fact that their market prices do not reflect their fundamental values. The key condition that allows for this kind of behavior is arbitrage-free pricing of all assets. If we use a model without micro-foundation and directly impose a no-arbitrage condition, as we do, then there is obviously no inconsistency with the usual interpretation of asset price bubbles. We therefore believe that it makes perfect sense to study this phenomenon also in the simpler Solow-Swan model, especially if this yields results (such as oscillations) which cannot be obtained in standard models that are based on micro-foundation. The Solow-Swan model has the additional advantage over the overlapping generations model that it can easily be formulated in continuous time, in which case diagrammatic analysis is especially simple and illuminating. ${ }^{1}$ A final reason for the use of a continuous-time SolowSwan model is that, due to its simplicity, it forms an especially challenging playing field for establishing the existence of oscillating or periodic equilibria. We address this challenge by using local stability analysis and the Hopf bifurcation theorem.

The outline of the paper is as follows. In Sect. 2 we briefly describe the framework of our analysis and summarize the known results on the dynamic (in)efficiency of the equilibria of the Solow-Swan model. Section 3 extends the standard Solow-Swan model by introducing an intrinsically useless asset and by assuming that consumption is proportional to GDP plus capital gains. Section 4 analyzes under which circumstances the second asset can coexist (with a positive price) with physical capital. We allow for positive and negative population growth rates and emphasize the differences between these two scenarios. Section 5 demonstrates that for negative population growth rates capital allocation paths in bubbly equilibria may display non-monotonic and perpetually oscillating behavior. In Sect. 6 we consider a version of the extended Solow-Swan model in which behavior is described by an investment rule rather than

\footnotetext{
1 Continuous-time formulations of overlapping generations models are of course available, but they either lead to infinite-dimensional aggregate dynamics or they require additional assumptions such as the perpetual youth model of Blanchard (1985) and Yaari (1965). Tirole (1985, p. 1077) also displays a phase diagram as an illustration but warns that "it is not meant to be a substitute for the discrete time analysis".
} 
by a consumption rule. In this case, permanently oscillating bubbles can also occur if the population growth rate is positive and the standard Solow-Swan equilibrium is dynamically efficient. Finally, Sect. 7 concludes the paper and outlines questions for future research.

\section{A one-sector economy, dynamic (in)efficiency, and the Solow-Swan model}

In this section we briefly review the framework of our analysis and discuss under which conditions equilibria are dynamically efficient or not.

Consider a one-sector economy that evolves in continuous time over the infinite time horizon $\mathbb{R}_{+}$. The population is assumed to grow at the constant rate $n$ and its initial size is normalized to be equal to 1 . The population size at time $t \in \mathbb{R}_{+}$is therefore given by $e^{n t}$. There exists a single production sector that transforms the input factors capital and labor into an output good which can be consumed or invested. We denote the intensive production function by $F: \mathbb{R}_{+} \mapsto \mathbb{R}_{+}$and assume that $F$ is a continuous and strictly increasing function satisfying $F(0)=0$. We assume furthermore that $F$ is twice continuously differentiable on the interior of its domain, that the Inada conditions $\lim _{k \rightarrow 0} F^{\prime}(k)=+\infty$ and $\lim _{k \rightarrow+\infty} F^{\prime}(k)=0$ are satisfied, and that $F^{\prime \prime}(k)<0$ holds for all $k>0$. Finally, we assume that capital depreciates at the constant rate $\delta$. We assume that both $\delta$ and $\delta+n$ are strictly positive numbers, but we do not require the population growth rate to be positive. It follows from the above assumptions that there exists a unique value $K>0$ satisfying $F(K)=(\delta+n) K$. This value $K$ is the maximal sustainable per capita capital stock. The initial (per capita) capital endowment of the economy is denoted by $\kappa$ and it is assumed that $\kappa \in(0, K)$.

We denote by $k(t), c(t)$, and $i(t)$ the per capita capital stock, the per capita consumption rate, and the per capita investment rate at time $t \in \mathbb{R}_{+}$. Note that the functions $k$, $c$, and $i$ are defined on the time domain $\mathbb{R}_{+}$and take values in $\mathbb{R}$. The triple $(k, c, i)$ is called a feasible allocation, if $c$ and $i$ are measurable, if $k$ is absolutely continuous, and if the conditions

$$
\begin{aligned}
\dot{k}(t) & =i(t)-(\delta+n) k(t), \quad k(0)=\kappa, \\
c(t)+i(t) & =F(k(t)), \\
c(t) & \geq 0, \quad k(t) \geq 0
\end{aligned}
$$

hold for almost all $t \in \mathbb{R}_{+}$. The first equation is the capital accumulation equation which says that net investment equals gross investment minus depreciation. The second one can be interpreted as the output market clearing condition and reflects the fact that final output can be used for consumption or for investment. The third line imposes nonnegativity of consumption and capital at all times. Note that investment is not required to be non-negative, which implies that output that has been installed as capital can be turned into the consumption good.

A feasible allocation $(k, c, i)$ is called dynamically inefficient if there exists another feasible allocation $(\tilde{k}, \tilde{c}, \tilde{i})$ such that $\tilde{c}(t) \geq c(t)$ holds for almost all $t \in \mathbb{R}_{+}$and such 
that the set $\left\{t \in \mathbb{R}_{+} \mid \tilde{c}(t)>c(t)\right\}$ has positive measure. A feasible triple $(k, c, i)$ is called dynamically efficient if it is not dynamically inefficient. Translating the conditions derived by Cass (1972) into the continuous-time setting of the present paper it follows that a feasible allocation $(k, c, i)$ is dynamically inefficient if and only if

$$
\lim _{\tau \rightarrow+\infty} \int_{0}^{\tau} R(t) \mathrm{d} t<+\infty
$$

holds, where

$$
R(t)=e^{\int_{0}^{t}\left[F^{\prime}(k(\tau))-(\delta+n)\right] \mathrm{d} \tau}
$$

The Golden Rule per capita capital stock $\bar{k}$ is defined as the unique positive number satisfying $F^{\prime}(\bar{k})=\delta+n$. Note that $\bar{k} \in(0, K)$ holds under the maintained assumptions. The efficiency criterion of Cass (1972) stated above gives rise to the following proposition, which will be useful for our analysis. ${ }^{2}$

Proposition 1 Let $(k, c, i)$ be afeasible allocation and assume that $k_{\infty}=\lim _{t \rightarrow+\infty} k(t)$ exists.

(a) If $k_{\infty}<\bar{k}$ holds, then it follows that $(k, c, i)$ is dynamically efficient.

(b) If $k_{\infty}>\bar{k}$ holds, then it follows that $(k, c, i)$ is dynamically inefficient.

(c) If $k_{\infty}=\bar{k}$ holds and if there exist positive numbers $M$ and $\mu$ such that $|k(t)-\bar{k}| \leq$ $M e^{-\mu t}$, then it follows that $(k, c, i)$ is dynamically efficient.

Proof (a) If $k_{\infty}<\bar{k}$, then it follows that $F^{\prime}\left(k_{\infty}\right)>F^{\prime}(\bar{k})=\delta+n$. Because of $\lim _{\tau \rightarrow+\infty} k(\tau)=k_{\infty}$, there exist $\varepsilon>0$ and $\tau_{\varepsilon}>0$ such that $F^{\prime}(k(\tau))-(\delta+n)>$ $\varepsilon>0$ holds for all $\tau>\tau_{\varepsilon}$. Obviously, this rules out that the limit in (4) is finite and it follows therefore that the allocation $(k, c, i)$ is dynamically efficient.

(b) Analogously to case (a) one can see that there exist $\varepsilon>0$ and $\tau_{\varepsilon}>0$ such that $F^{\prime}(k(\tau))-(\delta+n)<-\varepsilon<0$ holds for all $\tau>\tau_{\varepsilon}$. This implies that there exists a constant $S>0$ such that

$$
e^{\int_{0}^{t}\left[F^{\prime}(k(\tau))-(\delta+n)\right] \mathrm{d} \tau} \leq S e^{-(\varepsilon / 2) t} .
$$

Obviously, this makes the limit in (4) finite and it follows that $(k, c, i)$ is dynamically inefficient.

(c) Exponentially fast convergence of $k(t)$ to $\bar{k}$ implies that $F^{\prime}(k(\tau))-(\delta+n)$ converges exponentially fast to 0 . This property, in turn, shows that $\int_{0}^{t}\left[F^{\prime}(k(\tau))-\right.$ $(\delta+n)] \mathrm{d} \tau$ does not diverge to $-\infty$ as $t$ approaches $\infty$. This implies that $e^{\int_{0}^{t}\left[F^{\prime}(k((\tau))-(\delta+n)] \mathrm{d} \tau\right.}$ does not converge to 0 and it follows that $(4)$ is violated. Consequently, $(k, c, i)$ must be dynamically efficient.

For the hairline case $k_{\infty}=\bar{k}$ we have stated a sufficient efficiency condition only, which covers all cases occurring in this paper. This condition, which requires

$\overline{2}$ The result is widely known. We include the formal proof for completeness. 
exponentially fast convergence of $k(t)$ to the Golden Rule per capita capital stock $\bar{k}$, is not necessary for dynamic efficiency. On the other hand, convergence of $k(t)$ to $\bar{k}$ alone is not sufficient for dynamic efficiency. For example, if $k(t)$ approaches $\bar{k}$ slowly enough from above, the allocation $(k, c, i)$ can be dynamically inefficient.

The Solow-Swan model developed by Solow (1956) and Swan (1956) [see also Acemoglu (2009, chapter 2)] complements Eqs. (1)-(3) by a behavioral assumption that pins down the allocation of output between consumption and investment. Denoting by $s \in(0,1)$ the saving rate of the economy, this assumption can be formalized as

$$
c(t)=(1-s) F(k(t))
$$

Note that conditions (1)-(2) together with (5) imply that (3) is satisfied. Furthermore, it is worth mentioning that replacing the consumption rule (5) by the investment rule $i(t)=s F(k(t))$ leads to a completely equivalent model. Eliminating $c(t)$ and $i(t)$ from (1) to (2) and (5) it follows that

$$
\dot{k}(t)=s F(k(t))-(\delta+n) k(t), \quad k(0)=\kappa .
$$

Under the stated assumptions, this initial value problem has a unique solution which will be referred to as the standard Solow-Swan equilibrium. In this equilibrium the per capita capital stock $k(t)$ converges monotonically and exponentially fast to the unique positive solution of the equation $s F\left(k^{*}\right)=(\delta+n) k^{*}$. In other words, $k^{*} \in(0, K)$ is a globally asymptotically stable fixed point of (6). It holds furthermore that

$$
s F(k)-(\delta+n) k \begin{cases}>0 & \text { if } k<k^{*} \\ =0 & \text { if } k=k^{*}, \\ <0 & \text { if } k>k^{*},\end{cases}
$$

and that

$$
s F^{\prime}\left(k^{*}\right)<\delta+n \text {. }
$$

Note that $k^{*}$ can be smaller or larger than the Golden Rule per capita capital stock $\bar{k}$. It follows from Proposition 1 that the standard Solow-Swan equilibrium is dynamically inefficient if and only if $k^{*}>\bar{k}$.

\section{Introducing a second asset}

In the present section we modify the Solow-Swan model from the previous section by introducing a second asset, which is intrinsically useless: it cannot be consumed, it cannot be used in production, and it does not pay any dividends. This asset is available in constant supply, which we normalize by 1 . The price of the asset at time $t$ (in terms of contemporaneous consumption) will be denoted by $p(t)$ and it is assumed that

$$
\frac{\dot{p}(t)}{p(t)}=F^{\prime}(k(t))-\delta
$$


holds whenever $p(t)$ is positive. The left-hand side of (9) is the real return on the useless asset, while the right-hand side is the real return on physical capital. Condition (9) is therefore a no-arbitrage condition that ensures that physical capital and the useless asset have the same return. Any investor who cares only about the returns of assets would be indifferent between these two assets. ${ }^{3}$

Equations (1)-(2) from the original Solow-Swan model remain unchanged. Instead of the behavioral assumption (5), however, we now assume that the economy consumes the constant fraction $1-s$ of the sum of production income (GDP) and the appreciation of asset holdings. Taking into account that consumption has to remain non-negative we can express this consumption rule in per capita terms as

$$
c(t)=\max \left\{(1-s)\left[F(k(t))+\dot{p}(t) e^{-n t}\right], 0\right\} .
$$

Equation (10) is the key assumption of the model and generates a feedback from asset price movements to the allocation of output between consumption and investment. Finally, we need to explicitly impose the requirement that the aggregate capital stock must remain non-negative, that is,

$$
k(t) \geq 0 .
$$

The five equations (1)-(2) and (9)-(11) constitute the extended Solow-Swan model. It will be convenient to reformulate this model as a system of two autonomous differential equations. To this end let us define the variable $q(t)=p(t) e^{-n t}$. With this definition one can rewrite the no-arbitrage condition (9) as

$$
\dot{q}(t)=\left[F^{\prime}(k(t))-(\delta+n)\right] q(t) .
$$

Moreover, the consumption rule (10) can be expressed as

$$
c(t)=\left\{\begin{array}{cl}
(1-s)\left\{F(k(t))+\left[F^{\prime}(k(t))-\delta\right] q(t)\right\} & \text { if } F(k(t))+\left[F^{\prime}(k(t))-\delta\right] q(t) \geq 0, \\
0 & \text { otherwise }
\end{array}\right.
$$

Let us define the phase space $P=[0, K] \times \mathbb{R}_{+}$and the subset of the phase space on which consumption is strictly positive by

$$
P_{+}=\left\{(k, q) \mid k \in[0, K], q \geq 0, F(k)+\left[F^{\prime}(k)-\delta\right] q>0\right\} .
$$

We define $k_{\delta}$ as the unique value satisfying $F^{\prime}\left(k_{\delta}\right)=\delta$. Note that $k_{\delta}$ need not be contained in the interval $[0, K]$. If $k_{\delta} \geq K$, then it is clear that $P_{+}=P$. If $k_{\delta}<K$, however, then we have

$$
P_{+}=\left\{(k, q) \mid 0 \leq k \leq k_{\delta}, 0 \leq q\right\} \cup\left\{(k, q) \mid k_{\delta}<k \leq K, 0 \leq q<-F(k) /\left[F^{\prime}(k)-\delta\right]\right\} .
$$

\footnotetext{
3 In a micro-founded model in which the asset demand is derived from portfolio optimization problems, condition (9) would have to hold in any equilibrium in which both assets are held and traded. In the present model, which lacks micro-foundation, the condition cannot be derived but has to be assumed.
} 
Using (2) to eliminate $i(t)$ from (1) and substituting the consumption rule stated above for $c(t)$, one obtains the differential equation

$$
\dot{k}(t)= \begin{cases}s F(k(t))-(\delta+n) k(t)-(1-s)\left[F^{\prime}(k(t))-\delta\right] q(t) & \text { if }(k(t), q(t)) \in P_{+}, \\ F(k(t))-(\delta+n) k(t) & \text { otherwise, }\end{cases}
$$

with the initial condition $k(0)=\kappa$.

If $q(0)=0$ is satisfied, then it follows from (12) that $q(t)=0$ holds for all $t \in \mathbb{R}_{+}$ and Eq. (13) boils down to the standard Solow-Swan model (6). We formulate this observation in the following proposition.

Proposition 2 There exists a unique solution of conditions (11)-(13) which satisfies $q(t)=0$ for all $t \in \mathbb{R}_{+}$. This solution is the standard Solow-Swan equilibrium in which $k(t)$ converges to $k^{*}$.

\section{Asset price bubbles}

In this section we investigate under which conditions there exist solutions of the extended Solow-Swan model (11)-(13) for which $q(0)$ is positive. It follows from Eq. (12) that in any such solution $q(t)>0$ holds for all $t \in \mathbb{R}_{+}$. We shall refer to these solutions as equilibria of the Solow-Swan model that involve asset price bubbles or simply as bubbly equilibria.

We use a phase diagram analysis in the $(k, q)$-plane to address this question. The relevant phase space is $P$. To simplify the analysis we will not consider those parameter constellations which lead to the hairline cases $k^{*}=\bar{k}, k^{*}=k_{\delta}$, or $\bar{k}=k_{\delta}$. Note, in particular, that $\bar{k} \neq k_{\delta}$ implies that $n \neq 0$. Furthermore, we remind the reader that $\delta+n>0$ is assumed throughout the paper and that $k^{*} \in(0, K)$ and $\bar{k} \in(0, K)$ hold, whereas $k_{\delta}$ can be smaller or larger than $K$.

According to Eq. (12), the isocline $\dot{q}(t)=0$ consists of two branches, namely $\{(k, q) \mid k \in[0, K], q=0\}$ and $\{(k, q) \mid k=\bar{k}, q \geq 0\}$. The former branch forms part of the horizontal axis of the $(k, q)$-phase diagram, whereas the latter one is a vertical line at $k=\bar{k}$.

Let us denote the isocline $\dot{k}(t)=0$ by $I$, that is, $I=\{(k(t), q(t)) \mid \dot{k}(t)=0\}$, where $\dot{k}(t)$ is specified in (13). If $k<k_{\delta}$, then it holds for all $q \geq 0$ that $(k, q) \in P_{+}$ and it follows from (13) that $(k, q) \in I$ is equivalent to

$$
q=\frac{s F(k)-(\delta+n) k}{(1-s)\left[F^{\prime}(k)-\delta\right]}
$$

If $k=k_{\delta}$, then $(k, q) \in I$ implies $s F(k)=(\delta+n) k$ and, hence, $k=k^{*}$. Since the hairline case $k_{\delta}=k^{*}$ has been ruled out, the isocline $I$ cannot contain any point of the form $\left(k_{\delta}, q\right)$. Finally, if $k>k_{\delta}$, then we have to distinguish two cases. If 
$(k, q) \in P_{+} \cap I$, then (14) has to hold together with

$$
\frac{s F(k)-(\delta+n) k}{(1-s)\left[F^{\prime}(k)-\delta\right]}<-\frac{F(k)}{F^{\prime}(k)-\delta} .
$$

The latter condition is equivalent to $F(k)>(\delta+n) k$ which, in turn, holds for all $k \in(0, K)$. Alternatively, if $(k, q) \notin P_{+}$but $(k, q) \in I$, then it follows that $F(k)=(\delta+n) k$ and, hence, $k=K$ and $q \geq-F(K) /\left[F^{\prime}(K)-\delta\right]$. To summarize, the isocline $\dot{k}(t)=0$ is given by

$$
I= \begin{cases}I_{+} & \text {if } k_{\delta} \geq K, \\ I_{+} \cup\left\{(K, q) \mid q \geq-F(K) /\left[F^{\prime}(K)-\delta\right]\right\} & \text { if } k_{\delta}<K,\end{cases}
$$

where

$$
I_{+}=\left\{(k, q) \mid \text { Eq. (14) holds, } k \in[0, K] \backslash\left\{k_{\delta}\right\} \text {, and } q \geq 0\right\} \text {. }
$$

The fixed points of system (12)-(13) are the intersections of the two isoclines. One such intersection is obviously given by the origin $(0,0)$. This trivial fixed point will not play any role in the following analysis. Another fixed point is $\left(k^{*}, 0\right)$ and it is easily seen that this is the only fixed point of (12)-(13) that satisfies $k>0$ and $q=0$. A third potential fixed point is $(\bar{k}, \bar{q})$ with

$$
\bar{q}=\frac{s F(\bar{k})-(\delta+n) \bar{k}}{(1-s) n} .
$$

This fixed point exists (in $P$ ) if and only if the sign of $s F(\bar{k})-(\delta+n) \bar{k}$ coincides with the sign of $n$. In other words, the fixed point $(\bar{k}, \bar{q})$ exists either if $n>0$ and $\bar{k}<k^{*}$ or if $n<0$ and $\bar{k}>k^{*}$. All three fixed points are located in $P_{+}$such that we do not need to consider the second line of (13) for analyzing the local stability of any of the fixed points.

The Jacobian matrix of system (12)-(13) evaluated at the fixed points $\left(k^{*}, 0\right)$ and $(\bar{k}, \bar{q})$ is given by

$$
J^{*}=\left(\begin{array}{cc}
s F^{\prime}\left(k^{*}\right)-(\delta+n) & -(1-s)\left[F^{\prime}\left(k^{*}\right)-\delta\right] \\
0 & F^{\prime}\left(k^{*}\right)-(\delta+n)
\end{array}\right)
$$

and

$$
\bar{J}=\left(\begin{array}{cc}
-(1-s)\left[\delta+n+F^{\prime \prime}(\bar{k}) \bar{q}\right] & -(1-s) n \\
F^{\prime \prime}(\bar{k}) \bar{q} & 0
\end{array}\right),
$$

respectively. Since $J^{*}$ is an upper triangular matrix, its eigenvalues coincide with its diagonal elements. From (8) it follows that the eigenvalue corresponding to the upper left diagonal element of $J^{*}$ is negative. The second eigenvalue of $J^{*}$ is negative if $k^{*}>\bar{k}$ and it is positive if $k^{*}<\bar{k}$. Hence, $\left(k^{*}, 0\right)$ is a stable node if $k^{*}>\bar{k}$ and it 
is a saddle point if $k^{*}<\bar{k}$. In the latter case, the vector $(1,0)^{\top}$ is easily seen to be an eigenvector corresponding to the negative eigenvalue, which shows that the stable manifold at the saddle point $\left(k^{*}, 0\right)$ is horizontal. Scrutinizing equations (12)-(13) it is clear that this stable manifold is given by $\{(k, q) \mid 0 \leq k \leq K, q=0\}$.

Turning to the potential fixed point $(\bar{k}, \bar{q})$ we see from (17) that the determinant of $\bar{J}$ is given by

$$
\bar{D}=(1-s) n F^{\prime \prime}(\bar{k}) \bar{q},
$$

which is negative for $n>0$ and positive for $-\delta<n<0$ (recall that $\bar{q}>0$ must hold for $(\bar{k}, \bar{q})$ to be located in $P)$. This implies that $(\bar{k}, \bar{q})$ is a saddle point whenever $n>0$ and that it is a node or a focus when $n<0$. In the latter case, the local stability of $(\bar{k}, \bar{q})$ depends on the sign of the trace of $\bar{J}$, which is given by

$$
\bar{T}=-(1-s)\left[\delta+n+F^{\prime \prime}(\bar{k}) \bar{q}\right] .
$$

More specifically, $(\bar{k}, \bar{q})$ is locally stable if $\bar{T}<0$. Because of (15) this is the case if and only if

$$
F^{\prime \prime}(\bar{k})>-\frac{(1-s) n(\delta+n)}{s F(\bar{k})-(\delta+n) \bar{k}} .
$$

If (20) holds with the opposite inequality sign, then it follows that $(\bar{k}, \bar{q})$ is unstable.

After these preparatory remarks, we formulate the main results of this section. We distinguish between the two cases $n>0$ and $-\delta<n<0$.

Theorem 1 Consider the extended Solow-Swan model (11)-(13) and suppose that $n>0$ holds.

(a) If $k^{*}<\bar{k}$ is satisfied, then the standard Solow-Swan equilibrium is the only equilibrium of the economy and it is dynamically efficient.

(b) If $k^{*}>\bar{k}$ is satisfied, then the standard Solow-Swan equilibrium is dynamically inefficient and there exist other equilibria, which involve asset price bubbles.

$\left(\mathrm{b}_{1}\right)$ There is a unique dynamically efficient bubbly equilibrium. In this equilibrium it holds that $\lim _{t \rightarrow+\infty}(k(t), q(t))=(\bar{k}, \bar{q})$.

$\left(\mathrm{b}_{2}\right)$ There exists a continuum of dynamically inefficient bubbly equilibria, which satisfy $\lim _{t \rightarrow+\infty}(k(t), q(t))=\left(k^{*}, 0\right)$.

Proof First note that $n>0$ implies $\delta+n>\delta$ and, hence, $\bar{k}<k_{\delta}$.

(a) In this case it holds that $k^{*}<\bar{k}<k_{\delta}$. As has been shown above, the isocline $\dot{k}(t)=0$ is given by (14) plus potentially a vertical line at the boundary of $P$, and it exists for all $k \in\left[0, k^{*}\right] \cup\left(k_{\delta}, K\right]$. This isocline intersects the horizontal axis at $k=k^{*}$ and, because of $k^{*}<\bar{k}<k_{\delta}$, it does not intersect the line $k=\bar{k}$. Thus, the two isoclines $\dot{k}(t)=0$ and $\dot{q}(t)=0$ have a unique non-trivial intersection at $\left(k^{*}, 0\right)$ (in addition to the trivial intersection at the origin). The phase diagram for this case is shown in Fig. 1. Because of $k^{*}<\bar{k}$, it follows that $\left(k^{*}, 0\right)$ is a saddle point. As we 


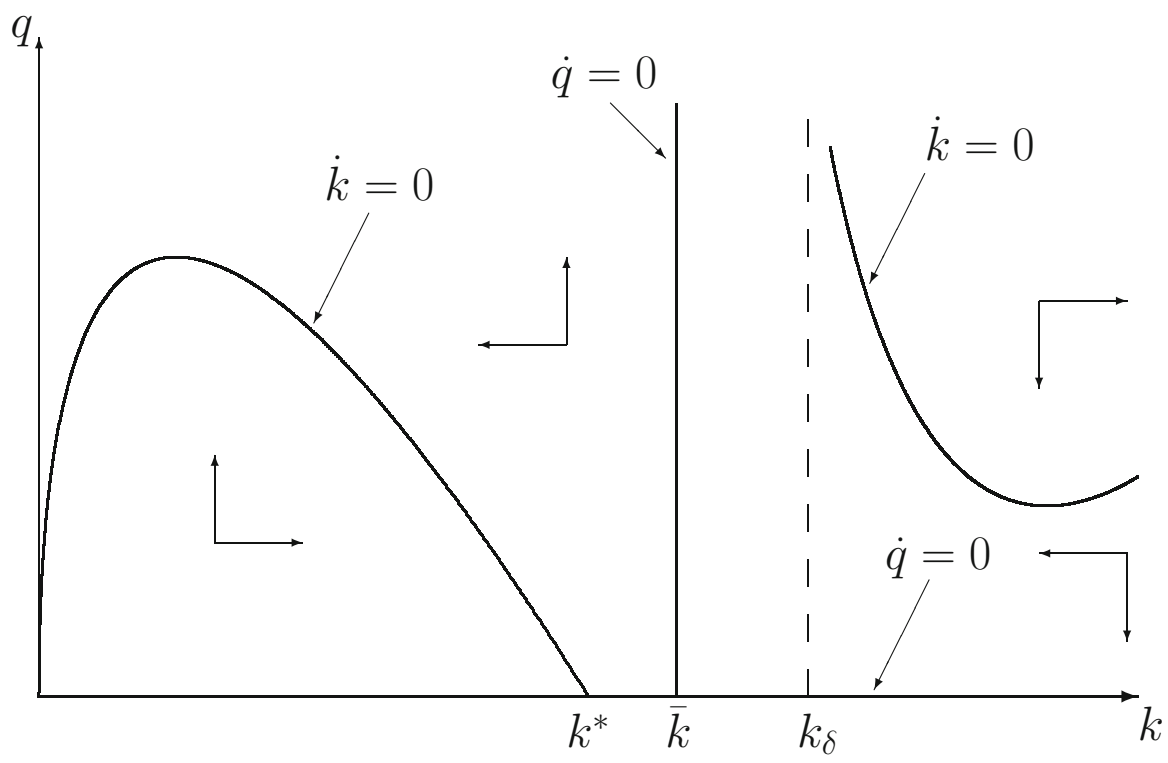

Fig. 1 The phase diagram for the case $n>0$ and $k^{*}<\bar{k}$ in Theorem 1(a)

have seen above, the stable manifold coincides with the horizontal axis. To the right of the vertical branch of the isocline $\dot{q}(t)=0, q(t)$ is decreasing and therefore cannot become unbounded. To the left of the vertical branch of the isocline $\dot{q}(t)=0$, on the other hand, unbounded growth of $q(t)$ is only possible by violating the constraint (11). This can be seen from $\mathrm{d} q / \mathrm{d} k=\dot{q}(t) / \dot{k}(t) \rightarrow-1 /(1-s)$ when $q(t)$ approaches $+\infty$ and $k(t) \in[0, \bar{k})$. Consequently, we conclude that the only solution of (11)-(13) is the stable saddle point path along which $\lim _{t \rightarrow+\infty} k(t)=k^{*}$ and $q(t)=0$ hold for all $t \in \mathbb{R}_{+}$. From Proposition 1 we know that this equilibrium is dynamically efficient.

(b) The case $k^{*}>\bar{k}$ consists of two subcases: $\bar{k}<k^{*}<k_{\delta}$ and $\bar{k}<k_{\delta}<k^{*}$. In both subcases there are two non-trivial intersections of the $\dot{k}(t)=0$ isocline specified by (14) and the $\dot{q}(t)=0$ isocline, namely $\left(k^{*}, 0\right)$ and $(\bar{k}, \bar{q})$. The phase diagram for the subcase $\bar{k}<k^{*}<k_{\delta}$ is shown in Fig. 2. Because of $\bar{k}<k^{*}$ the fixed point $\left(k^{*}, 0\right)$ is a stable node, and because of $n>0$ the fixed point $(\bar{k}, \bar{q})$ is a saddle point. The stable manifold of $(\bar{k}, \bar{q})$ is upward sloping and qualifies as an equilibrium involving a speculative bubble with $\lim _{t \rightarrow+\infty}(k(t), q(t))=(\bar{k}, \bar{q})$. According to Proposition 1 , this bubbly equilibrium is dynamically efficient. In addition, there exist infinitely many bubbly equilibria converging to $\left(k^{*}, 0\right)$. Referring again to Proposition 1 , these equilibria are dynamically inefficient. Using the same reasoning as in the proof of part (a) one can rule out that solutions of (12)-(13) for which $q(t)$ becomes unbounded satisfy the non-negativity constraint (11).

Consider now the subcase $\bar{k}<k_{\delta}<k^{*}$, for which the phase diagram is shown in Fig. 3. There are only a few differences to the previous subcase. As before there exist two fixed points (in addition to the trivial one), namely $\left(k^{*}, 0\right)$ and $(\bar{k}, \bar{q})$, whereby $\left(k^{*}, 0\right)$ is a stable node and $(\bar{k}, \bar{q})$ is a saddle point with an upward sloping stable 


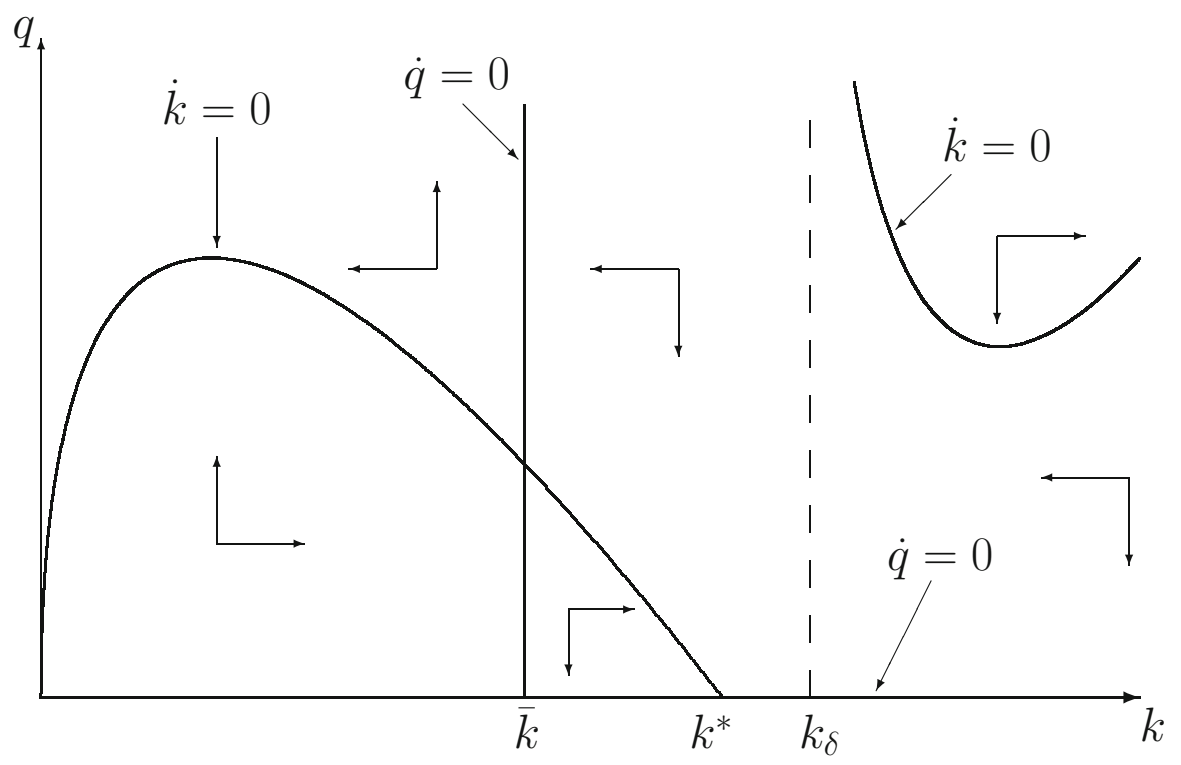

Fig. 2 The phase diagram for the case $n>0$ and $\bar{k}<k^{*}<k_{\delta}$ in Theorem 1(b)

manifold. Hence, the conclusions stated in part (b) of the theorem hold also true in this subcase.

Remark 1 Close examination of the phase diagrams reveals that some of the bubbly equilibria do not exist for all initial capital stocks $\kappa \in(0, K)$. As an example let us discuss the phase diagram for the case $\bar{k}<k_{\delta}<k^{*}$ shown in Fig. 3. The stable manifold of the saddle point $(\bar{k}, \bar{q})$ must be located in the two areas to the left of $k_{\delta}$ where $\dot{k}(t)$ and $\dot{q}(t)$ have the same sign. This implies obviously that it cannot extend beyond the asymptote of the isocline $\dot{k}(t)=0$ at $k=k_{\delta}$. As a consequence, the dynamically efficient bubbly equilibrium mentioned in theorem $1\left(b_{1}\right)$ exists only for initial capital endowments $\kappa \in\left(0, k_{\delta}\right)$. In the case $\bar{k}<k^{*}<k_{\delta}$, which is illustrated in Fig. 2, the situation is not so clear. Here it depends on whether the stable saddle point path of $(\bar{k}, \bar{q})$ lies below the right branch of the isocline $\dot{k}(t)=0$ or not. It does not seem to be possible to determine which of these two scenarios occurs without explicitly specifying the production function and the model parameters.

The results presented in theorem 1 correspond to those derived by Tirole (1985, proposition 1) for the overlapping generations model. In particular, one can see from the theorem that, under the assumption $n>0$, dynamic inefficiency of the standard Solow-Swan equilibrium is equivalent to the existence of asset price bubbles. We now turn to the case of a negative population growth rate $n$, which has no counterpart in Tirole (1985).

Theorem 2 Consider the extended Solow-Swan model (11)-(13) and suppose that $-\delta<n<0$ holds. 


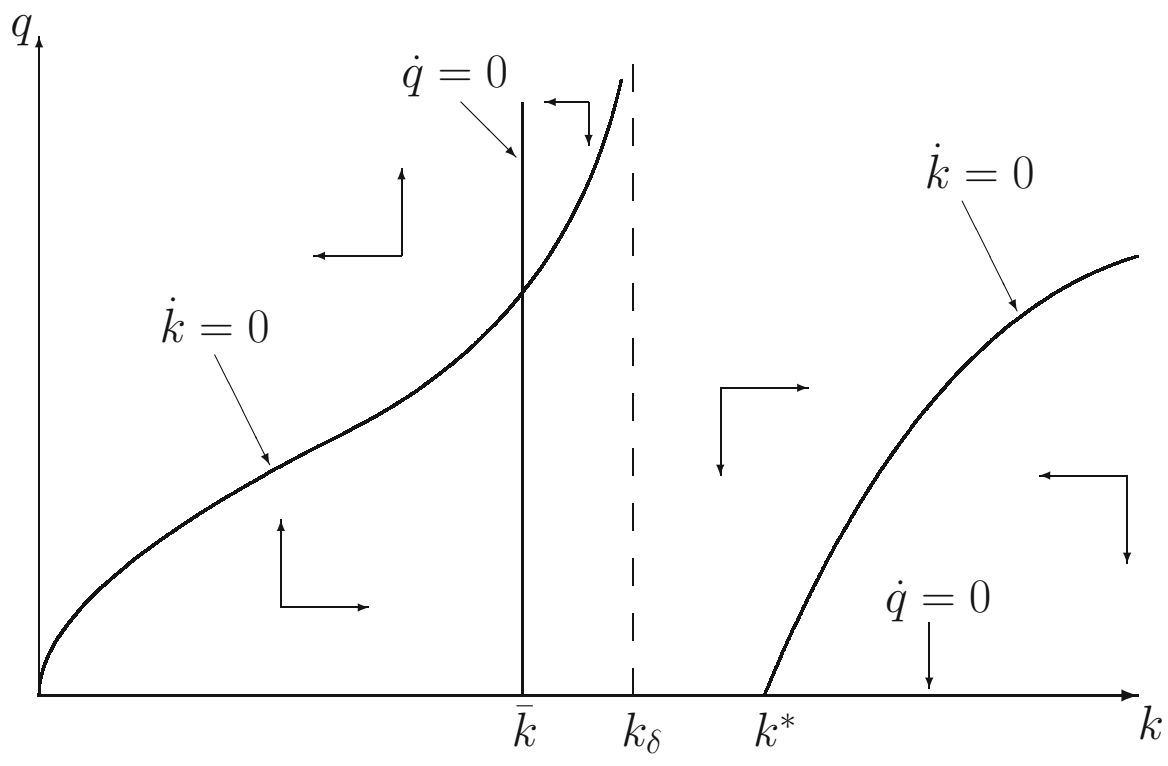

Fig. 3 The phase diagram for the case $n>0$ and $\bar{k}<k_{\delta}<k^{*}$ in Theorem 1(b)

(a) If $k^{*}<\bar{k}$ is satisfied, then the standard Solow-Swan equilibrium is dynamically efficient. Nevertheless, there can exist other dynamically efficient equilibria, which involve asset price bubbles and satisfy $\lim _{t \rightarrow+\infty}(k(t), q(t))=(\bar{k}, \bar{q})$. A sufficient condition for this to be the case is (20).

(b) If $k^{*}>\bar{k}$ is satisfied, then the standard Solow-Swan equilibrium is dynamically inefficient. There exist infinitely many dynamically inefficient bubbly equilibria with the property $\lim _{t \rightarrow+\infty}(k(t), q(t))=\left(k^{*}, 0\right)$.

Proof First note that $-\delta<n<0$ implies $0<\delta+n<\delta$ and, hence, $\bar{k}>k_{\delta}$.

(a) This case consists of the two subcases $k^{*}<k_{\delta}<\bar{k}$ and $k_{\delta}<k^{*}<\bar{k}$. The phase diagram for the subcase $k^{*}<k_{\delta}<\bar{k}$ is shown in Fig. 4. One can see that there are two non-trivial fixed points, namely $\left(k^{*}, 0\right)$ and $(\bar{k}, \bar{q})$. Because of $k^{*}<\bar{k}$, it follows that the fixed point $\left(k^{*}, 0\right)$ is a saddle point and that the stable manifold coincides with the horizontal axis. Now consider the Jacobian matrix $\bar{J}$ from (17). Determinant and trace of this matrix are given in (18) and (19), respectively, and one can see that they are related to each other by

$$
\bar{D}=-n[(1-s)(\delta+n)+\bar{T}] .
$$

Moreover, because of $n<0$, the determinant is positive. The $(\bar{T}, \bar{D})$-diagram is depicted in Fig. 5 and shows the locus $\left\{(\bar{T}, \bar{D}) \mid-\infty<F^{\prime \prime}(\bar{k})<0\right\}$. This locus is that part of the line specified in (21), which corresponds to negative values of $F^{\prime \prime}(\bar{k})$. More specifically, it starts at the point $(-(1-s)(\delta+n), 0)$ (corresponding to the limit as $F^{\prime \prime}(\bar{k})$ tends to 0$)$ and extends to the north-east as $F^{\prime \prime}(\bar{k})$ tends to $-\infty$. Hence, 


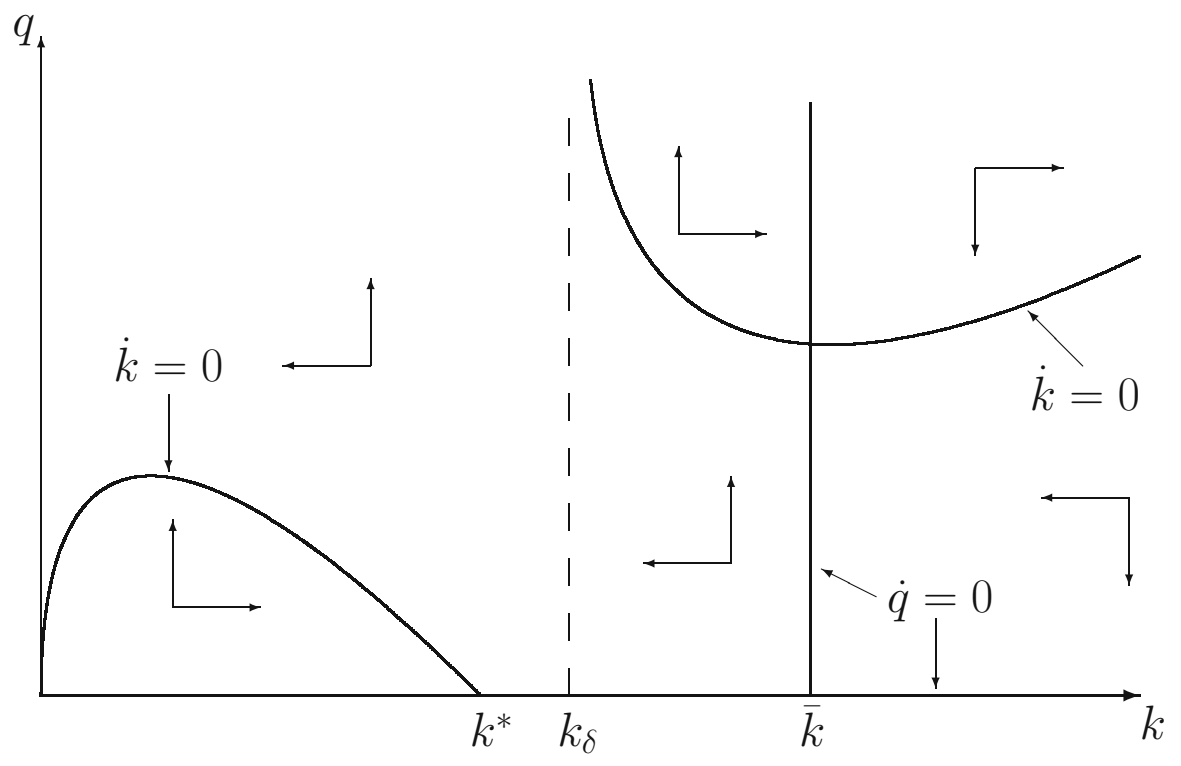

Fig. 4 The phase diagram for the case $-\delta<n<0$ and $k^{*}<k_{\delta}<\bar{k}$ in Theorem 2(a)

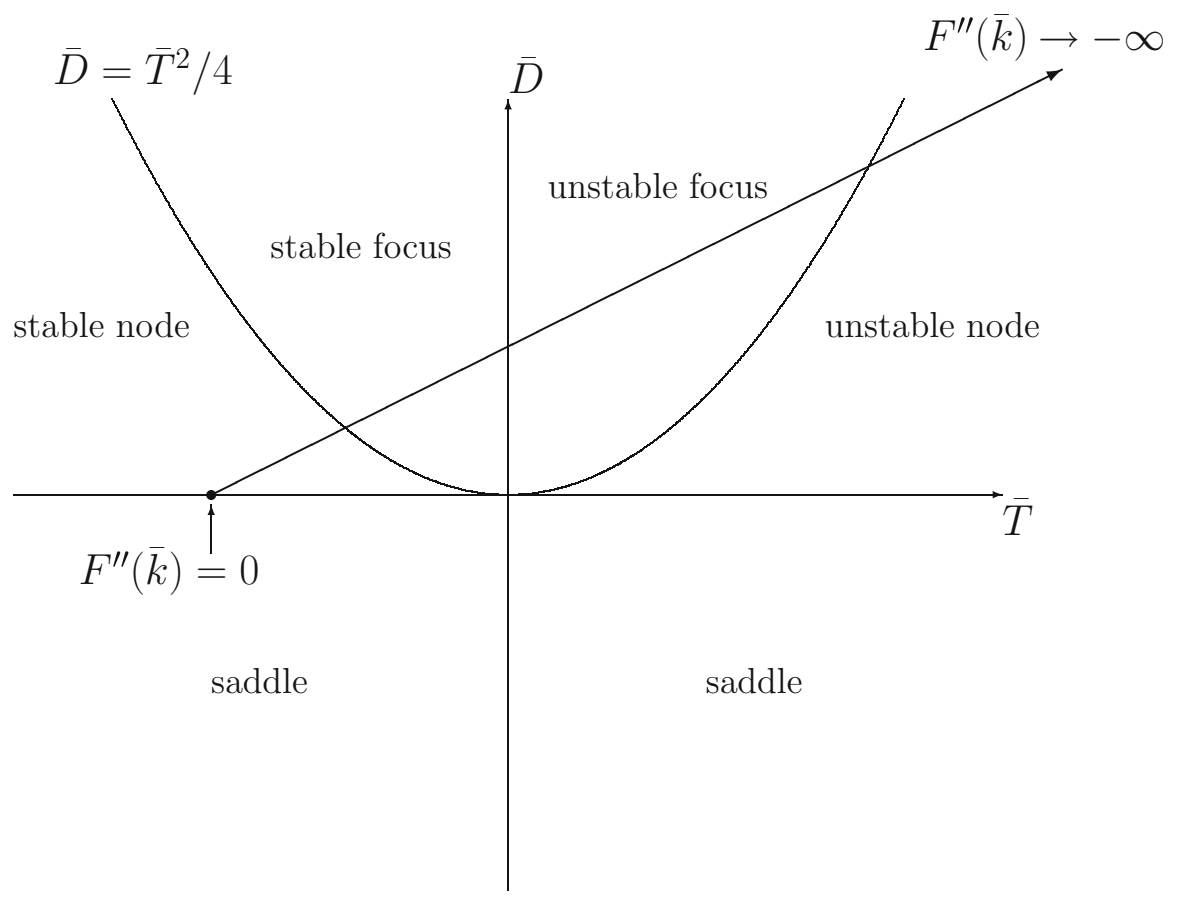

Fig. 5 The $(\bar{T}, \bar{D})$-diagram for the case $-\delta<n<0$ and $k^{*}<\bar{k}$ in Theorem 2(a) 


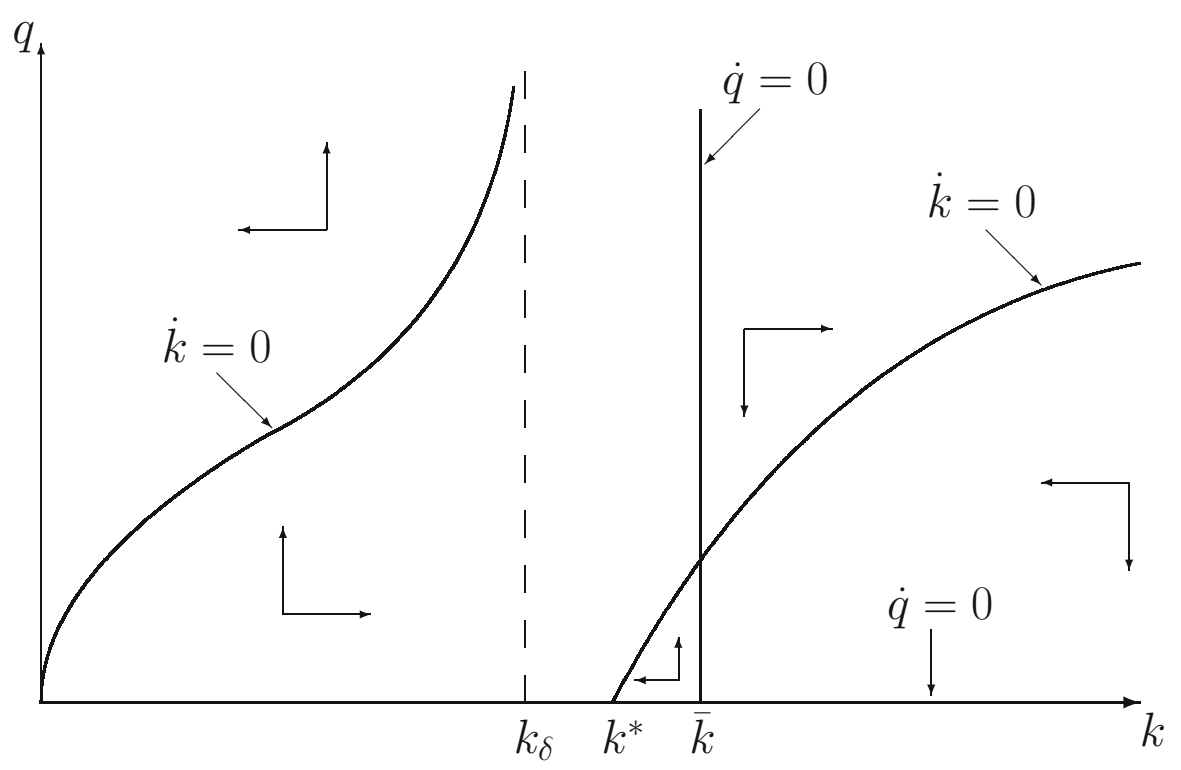

Fig. 6 The phase diagram for the case $-\delta<n<0$ and $k_{\delta}<k^{*}<\bar{k}$ in Theorem 2(a)

we conclude that $(\bar{k}, \bar{q})$ can be a stable node, a stable focus, an unstable focus, or an unstable node depending on the value of $F^{\prime \prime}(\bar{k})$. Whenever condition (20) holds, the trace $\bar{T}$ is negative and it follows that $(\bar{k}, \bar{q})$ is locally stable. In this case there exist infinitely many bubbly equilibria converging to $(\bar{k}, \bar{q})$. According to Proposition 1 , these equilibria are dynamically efficient. As in the proof of Theorem 1 one can rule out that there exist equilibria in which $q(t)$ becomes unbounded.

In the second subcase it holds that $k_{\delta}<k^{*}<\bar{k}$. The corresponding phase diagram is shown in Fig. 6. As in the previous subcase there exist two non-trivial fixed points $\left(k^{*}, 0\right)$ and $(\bar{k}, \bar{q})$, where $\left(k^{*}, 0\right)$ is a saddle point with a horizontal stable manifold. The Jacobian matrix $\bar{J}$ has the same trace and determinant as in the previous subcase and, hence, the stability properties of $(\bar{k}, \bar{q})$ are characterized by the very same conditions as before. In particular, the $(\bar{T}, \bar{D})$-diagram shown in Fig. 5 applies to this subcase as well. As before, there exist dynamically efficient bubbly equilibria with $\lim _{t \rightarrow+\infty}(k(t), q(t))=(\bar{k}, \bar{q})$ provided that (20) holds.

(b) In this case it holds that $k_{\delta}<\bar{k}<k^{*}$. The isocline $\dot{k}(t)=0$ has a pole at $k=k_{\delta}$ and exists only for $k \in\left[0, k_{\delta}\right) \cup\left[k^{*}, K\right]$. The phase diagram is shown in Fig. 7 and it is easily seen that the two isoclines have a unique intersection (except for the trivial one at the origin), which occurs at $\left(k^{*}, 0\right)$. Since $k^{*}>\bar{k}$ holds, it follows that $\left(k^{*}, 0\right)$ is a stable node. This means that there exist infinitely many equilibria involving asset price bubbles in addition to the standard Solow-Swan equilibrium. All these bubbly equilibria converge to $\left(k^{*}, 0\right)$ and, according to Proposition 1 , they are dynamically inefficient. It is also straightforward to see from the phase diagram that, except for those solutions of (11)-(13) that converge to $\left(k^{*}, 0\right)$, there do not exist any other solutions. 


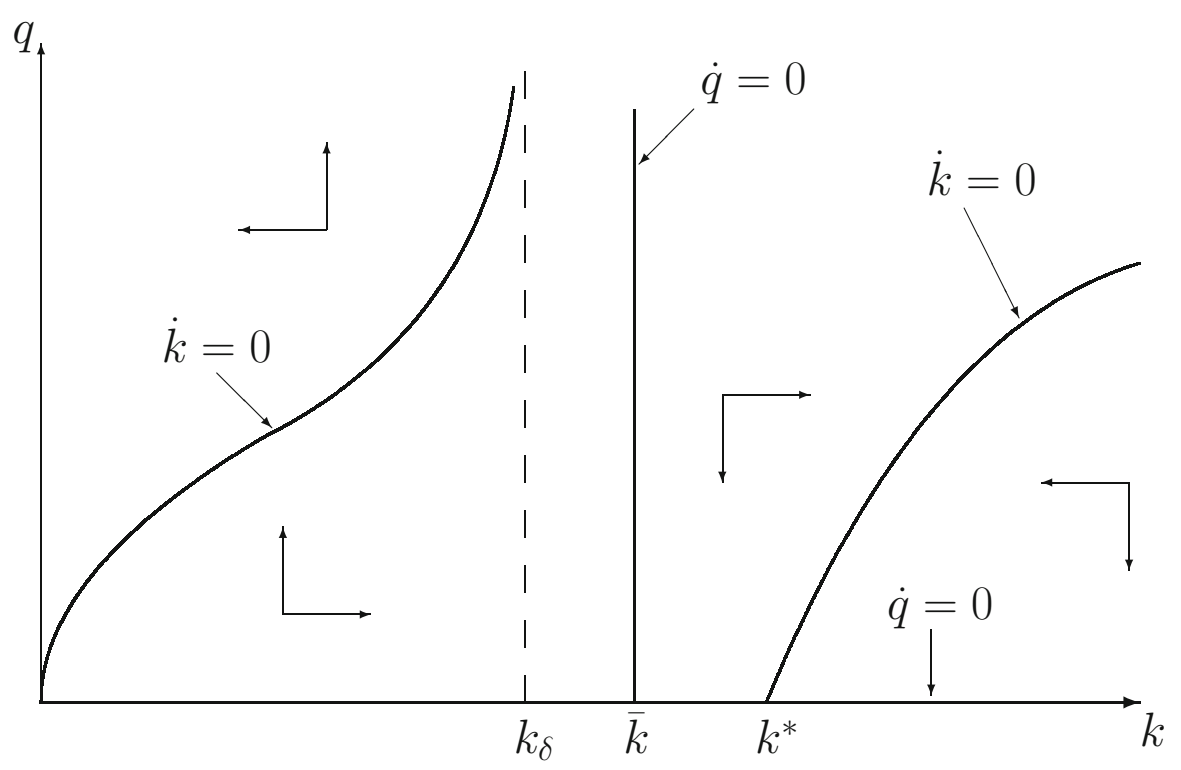

Fig. 7 The phase diagram for the case $-\delta<n<0$ and $k_{\delta}<\bar{k}<k^{*}$ in Theorem 2(b)

We can see from Theorem 2 that in the case $n<0$ dynamic efficiency of the standard Solow-Swan equilibrium (i.e., the assumption that $k^{*}<\bar{k}$ holds) does not rule out the existence of asset price bubbles, whereas it still holds that dynamic inefficiency of the standard Solow-Swan equilibrium implies the existence of bubbly equilibria. A comment similar to Remark 1 applies to Theorem 2 as well.

\section{Bubbly cycles}

In the present section we demonstrate that the asset price bubbles detected in the previous section can generate non-monotonic, oscillating, or even periodic capital accumulation paths.

A first instance of a non-monotonic path can be seen in the phase diagram in Fig. 2. Suppose that $k(0)=\kappa \in\left(\bar{k}, k^{*}\right)$ and $q(0)=\bar{q}$. It is easily seen from the phase diagram that the corresponding solution of (12)-(13) starts in south-west direction until it crosses the isocline $\dot{k}(t)=0$. From that point onwards, it continues in southeast direction. That is, this bubbly equilibrium exhibits first a decreasing per capita capital stock and then an increasing one. A similar phenomenon occurs in the phase diagrams in Figs. 3 and 7. Consider any trajectory in these figures that starts at a point $\left(k^{*}, q(0)\right)$ with $q(0)>0$. Such a trajectory moves to the south-east, crosses the isocline $\dot{k}(t)=0$, and continues in the south-west direction, which means that the per capita capital stock $k(t)$ first increases and then decreases until it approaches $k^{*}$.

In the examples from the previous paragraph, the capital accumulation paths are non-monotonic but they are eventually monotonic. In what follows we show that there 
can be permanent oscillations, too. Indeed, this is the case if $(\bar{k}, \bar{q})$ is a stable focus, which requires that the stability condition (20) is satisfied along with the condition $\bar{D}>\bar{T}^{2} / 4$. The latter condition ensures that the eigenvalues of $\bar{J}$ are complex numbers.

Corollary 1 Suppose that $-\delta<n<0, k^{*}<\bar{k}$, and (20) hold. If, in addition, the inequality

$$
\begin{aligned}
F^{\prime \prime}(\bar{k}) & <\frac{n}{s F(\bar{k})-(\delta+n) \bar{k}} \\
& \times\left[2 n-(1-s)(\delta+n)+2 \sqrt{n^{2}-(1-s) n(\delta+n)}\right]
\end{aligned}
$$

is satisfied, then it follows that the asset price bubbles described in Theorem 2(a) display permanent but damped oscillations.

Proof Let us define $\bar{z}=F^{\prime \prime}(\bar{k}) \bar{q}$. Using the expressions for $\bar{D}$ and $\bar{T}$ from (18) and (19), respectively, and the expression for $\bar{q}$ from (15) it follows that

$$
\bar{D}-\bar{T}^{2} / 4=\frac{1-s}{4}\left[4 n \bar{z}-(1-s)(\delta+n+\bar{z})^{2}\right] .
$$

Consequently, $\bar{D}>\bar{T}^{2} / 4$ is equivalent to $4 n \bar{z}>(1-s)(\delta+n+\bar{z})^{2}$. This inequality, in turn, holds for $z_{1}<\bar{z}<z_{2}$, where

$$
z_{1,2}=\frac{2 n-(1-s)(\delta+n) \pm 2 \sqrt{n^{2}-(1-s) n(\delta+n)}}{1-s}
$$

Condition (20) implies that $z_{1}<\bar{z}$ holds, whereas condition $\bar{z}<z_{2}$ is easily seen to be equivalent to (22).

Finally, we note that, as $F^{\prime \prime}(\bar{k})$ passes the threshold specified in (20), a Hopf bifurcation occurs at the fixed point $(\bar{k}, \bar{q})$ such that the system (12)-(13) has periodic solutions. The periodic solutions exist for values of $F^{\prime \prime}(\bar{k})$ smaller than the right-hand side of (20) if the Hopf bifurcation is super-critical, and they exist for values of $F^{\prime \prime}(\bar{k})$ larger than the right-hand side of (20) if the Hopf bifurcation is sub-critical; see, e.g., Guckenheimer and Holmes (1983, theorem 3.4.2). Proposition 1 cannot be used to study the dynamic efficiency of these periodic equilibria, but the Cass criterion stated in Sect. 2 could still be applied.

Example 1 Suppose that the production function is of Cobb-Douglas type $F(k)=k^{\alpha}$ with $\alpha \in(0,1)$. Straightforward calculations yield

$$
\bar{T}=(\delta+n)\left[s-1+\frac{(1-\alpha)(s-\alpha)(\delta+n)}{\alpha n}\right]
$$


and

$$
\begin{aligned}
\bar{D}-\frac{\bar{T}^{2}}{4} & =\frac{(\delta+n)^{2}}{4} \\
& \times\left\{4(1-\alpha)\left(1-\frac{s}{\alpha}\right)-\left[s-1+\frac{(1-\alpha)(s-\alpha)(\delta+n)}{\alpha n}\right]^{2}\right\} .
\end{aligned}
$$

If $-\delta<n<0$ holds, then it follows that the inequality $\bar{T}<0$ is equivalent to

$$
s[(1-\alpha) \delta+n]>\alpha[(1-\alpha)(\delta+n)+n] .
$$

This condition can also be written as

$$
\frac{n}{\delta} \leq-\frac{1-\alpha}{2-\alpha} \quad \text { or } \quad s>\alpha\left[1+\frac{(1-\alpha)(n / \delta)}{1-\alpha+(n / \delta)}\right] .
$$

If (25) holds, then $\bar{D}-\bar{T}^{2} / 4$ is positive if and only if

$$
s<\frac{\alpha\left[(1-\alpha)^{2}+(1-\alpha)(3-\alpha)(n / \delta)+\alpha(n / \delta)^{2}-2(1-\alpha)(n / \delta) \sqrt{-(1-\alpha)(n / \delta)}\right]}{[1-\alpha+(n / \delta)]^{2}} .
$$

Figure 8 shows the $(n / \delta, s)$-parameter space for the case where $\alpha=1 / 3$. In the top area of this figure it holds that $s>\alpha$, which is equivalent to $k^{*}>\bar{k}$. From Theorem 2(b) we know that in this parameter region there exist asset price bubbles converging to $\left(k^{*}, 0\right)$ but that the fixed point $(\bar{k}, \bar{q})$ is not located in $P$. In the parameter region $s<\alpha$ (or, equivalently, $k^{*}<\bar{k}$ ), the fixed point $(\bar{k}, \bar{q})$ exists. Theorem 2(a) tells us that bubbly equilibria converging to $(\bar{k}, \bar{q})$ occur provided that (20) holds which, in the present example, is equivalent to (25). In the figure, the parameter region in which (25) holds consists of the two areas labelled 'stable node' and 'stable focus'. According to Corollary 1 the asset price bubbles converging to $(\bar{k}, \bar{q})$ display damped oscillations whenever condition (22) is satisfied in addition to (20). In the present example, this means that (25) and (26) must hold simultaneously. The corresponding parameter region in Fig. 8 is labelled 'stable focus'. Finally, the bottom right part of the relevant parameter region, which is labelled as 'unstable', corresponds of those pairs $(n / \delta, s)$ for which the fixed point $(\bar{k}, \bar{q})$ is unstable. For parameter constellations in this area Theorem 2 is silent. The upper boundary of this area, however, is the locus where Hopf bifurcations occur. These bifurcations generate periodic asset price bubbles.

\section{A model with an investment rule}

In Sects. 3-5 we have considered an extension of the Solow-Swan model in which the consumption rule (5) is replaced by (10). Whereas in the standard Solow-Swan model 


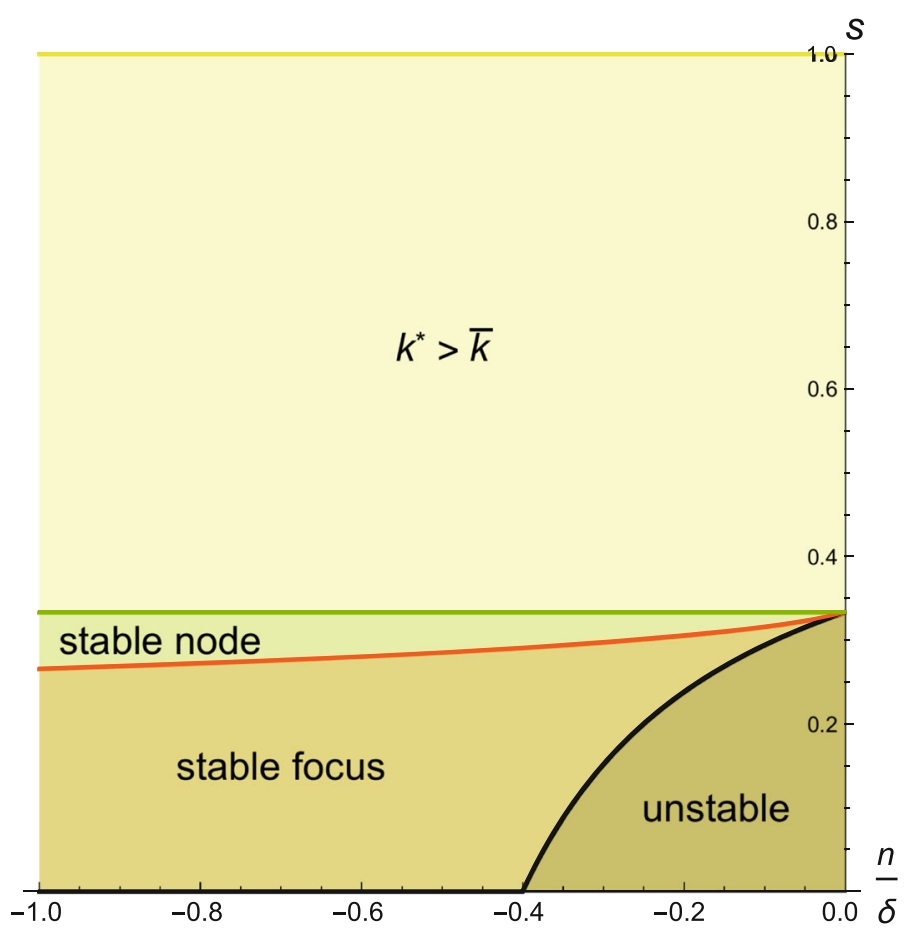

Fig. 8 The $(n / \delta, s)$-parameter space for Example 1

the consumption rule (5) is fully equivalent to the investment rule $i(t)=s F(k(t))$, this equivalence breaks down when the rules condition on total income (GDP plus capital gains) and not on GDP alone. The purpose of the present section is therefore to study equilibria in a model in which investment is a fixed fraction of total income.

Because of the output market clearing condition $c(t)+i(t)=F(k(t))$ and because consumption has to remain non-negative, the inequality $i(t) \leq F(k(t))$ has to be satisfied. The model under consideration consists therefore of the standard equations (1)-(2), the no-arbitrage condition (9), the non-negativity constraint (11), and the investment rule

$$
i(t)=\min \left\{s\left[F(k(t))+\dot{p}(t) e^{-n t}\right], F(k(t))\right\} .
$$

As in Sect. 3 we start by reformulating the model as a system of two autonomous differential equations in the variables $k(t)$ and $q(t)=p(t) e^{-n t}$. The no-arbitrage condition is the same as in the previous model, namely

$$
\dot{q}(t)=\left[F^{\prime}(k(t))-(\delta+n)\right] q(t) .
$$


The subset of the $(k, q)$-phase space $P$ on which consumption is strictly positive is now given by

$$
P_{+}=\left\{(k, q) \mid k \in[0, K], q \geq 0,(1-s) F(k)>s\left[F^{\prime}(k)-\delta\right] q\right\} .
$$

This set can also be written as $P_{+}=P_{+1} \cup P_{+2}$, where

$$
P_{+1}=\left\{(k, q) \mid k_{\delta} \leq k \leq K, 0 \leq q\right\}
$$

and

$$
P_{+2}=\left\{(k, q) \mid 0 \leq k \leq \min \left\{k_{\delta}, K\right\}, 0 \leq q<(1-s) F(k) /\left\{s\left[F^{\prime}(k)-\delta\right]\right\}\right\} .
$$

Substituting the investment rule (27) into the capital accumulation equation (1) we therefore obtain the differential equation

$$
\dot{k}(t)= \begin{cases}s F(k(t))-(\delta+n) k(t)+s\left[F^{\prime}(k(t))-\delta\right] q(t) & \text { if }(k(t), q(t)) \in P_{+}, \\ F(k(t))-(\delta+n) k(t) & \text { otherwise }\end{cases}
$$

with the initial condition $k(0)=\kappa$. The full model considered in the present section consists of equations (28)-(29) and the non-negativity constraint (11). The following proposition is the counterpart to Proposition 2 from Sect. 3.

Proposition 3 There exists a unique solution of conditions (11) and (28)-(29) for which $q(t)=0$ holds for all $t \in \mathbb{R}_{+}$. This is the standard Solow-Swan equilibrium in which $k(t)$ converges to $k^{*}$.

Before we characterize the conditions under which bubbly equilibria exist in the present version of the model, let us emphasize that the only difference to the model discussed in the previous sections consists of imposing the investment rule (27) instead of the consumption rule (10). According to the rule (27), investment is positively affected by an appreciation of the asset price $p(t)$, whereas the consumption rule (27) in combination with the feasibility condition (2) makes investment negatively dependent on $\dot{p}(t)$. This difference results in a negative coefficient in front of $\left[F^{\prime}(k(t))-\delta\right] q(t)$ in the reduced Eq. (13) and a positive one in the corresponding Eq. (29). Whether asset price movements have a direct effect on consumption demand or investment demand has important consequences that will be outlined in the rest of the present section.

The analysis of bubbly equilibria follows the same steps as in the preceding sections and we will therefore not provide all details. We carry out a phase diagram analysis of equations (28)-(29) in the phase space $P$. As before, we do not consider parameter constellations which lead to the hairline cases $k^{*}=\bar{k}, k^{*}=k_{\delta}$, or $\bar{k}=k_{\delta}$.

The isocline $\dot{q}(t)=0$ consists of two branches, namely $\{(k, q) \mid k \in[0, K], q=0\}$ and $\{(k, q) \mid k=\bar{k}, q \geq 0\}$. To determine the shape of the isocline $\dot{k}(t)=0$, which will be denoted by $I$, we observe that $(k, q) \in P_{+} \cap I$ implies

$$
q=-\frac{s F(k)-(\delta+n) k}{s\left[F^{\prime}(k)-\delta\right]}
$$


Defining

$$
I_{+}=\left\{(k, q) \mid \text { equation (30) holds, } k \in[0, K] \backslash\left\{k_{\delta}\right\} \text {, and } q \geq 0\right\}
$$

it follows that

$$
I=\left\{\begin{array}{cl}
I_{+} & \text {if } k_{\delta} \geq K, \\
I_{+} \cup\left\{(K, q) \mid q \geq(1-s) F(K) /\left\{s\left[F^{\prime}(K)-\delta\right]\right\}\right\} & \text { if } k_{\delta}<K,
\end{array}\right.
$$

System (28)-(29) has potentially three fixed points, namely $(0,0),\left(k^{*}, 0\right)$, and $(\bar{k}, \bar{q})$ with

$$
\bar{q}=-\frac{s F(\bar{k})-(\delta+n) \bar{k}}{s n} .
$$

The first one will not play any role for the analysis, the second one corresponds to the fixed point of the standard Solow-Swan model and exists under all parameter constellations, and the last one exists in $P$ if and only if $s F(\bar{k})-(\delta+n) \bar{k}$ has the opposite sign as $n$. In other words, the fixed point $(\bar{k}, \bar{q})$ exists either if $n>0$ and $\bar{k}>k^{*}$ or if $n<0$ and $\bar{k}<k^{*}$. The two non-trivial fixed points are located in $P_{+}$ such that we do not need to consider the second line of (29) for analyzing their local stability.

The Jacobian matrix of system (28)-(29) evaluated at the fixed points $\left(k^{*}, 0\right)$ and $(\bar{k}, \bar{q})$ is given by

$$
J^{*}=\left(\begin{array}{cc}
s F^{\prime}\left(k^{*}\right)-(\delta+n) & s\left[F^{\prime}\left(k^{*}\right)-\delta\right] \\
0 & F^{\prime}\left(k^{*}\right)-(\delta+n)
\end{array}\right)
$$

and

$$
\bar{J}=\left(\begin{array}{cc}
-(1-s)(\delta+n)+s F^{\prime \prime}(\bar{k}) \bar{q} & s n \\
F^{\prime \prime}(\bar{k}) \bar{q} & 0
\end{array}\right),
$$

respectively. The fixed point $\left(k^{*}, 0\right)$ is a stable node if $k^{*}>\bar{k}$ and it is a saddle point if $k^{*}<\bar{k}$. In the latter case, the stable manifold is given by $\{(k, q) \mid 0 \leq k \leq K, q=0\}$.

The determinant and the trace of $\bar{J}$ are given by

$$
\bar{D}=-\operatorname{sn} F^{\prime \prime}(\bar{k}) \bar{q}
$$

and

$$
\bar{T}=-(1-s)(\delta+n)+s F^{\prime \prime}(\bar{k}) \bar{q},
$$

respectively. Note that $\delta+n>0, \bar{q}>0$, and $F^{\prime \prime}(\bar{k})<0$ imply that $\bar{T}$ is negative for all parameter constellations. The determinant $\bar{D}$, however, is negative for $-\delta<n<0$ and positive for $n>0$. This implies that $(\bar{k}, \bar{q})$ is a saddle point whenever $-\delta<n<0$ and that it is a stable node or a stable focus when $n>0$. 


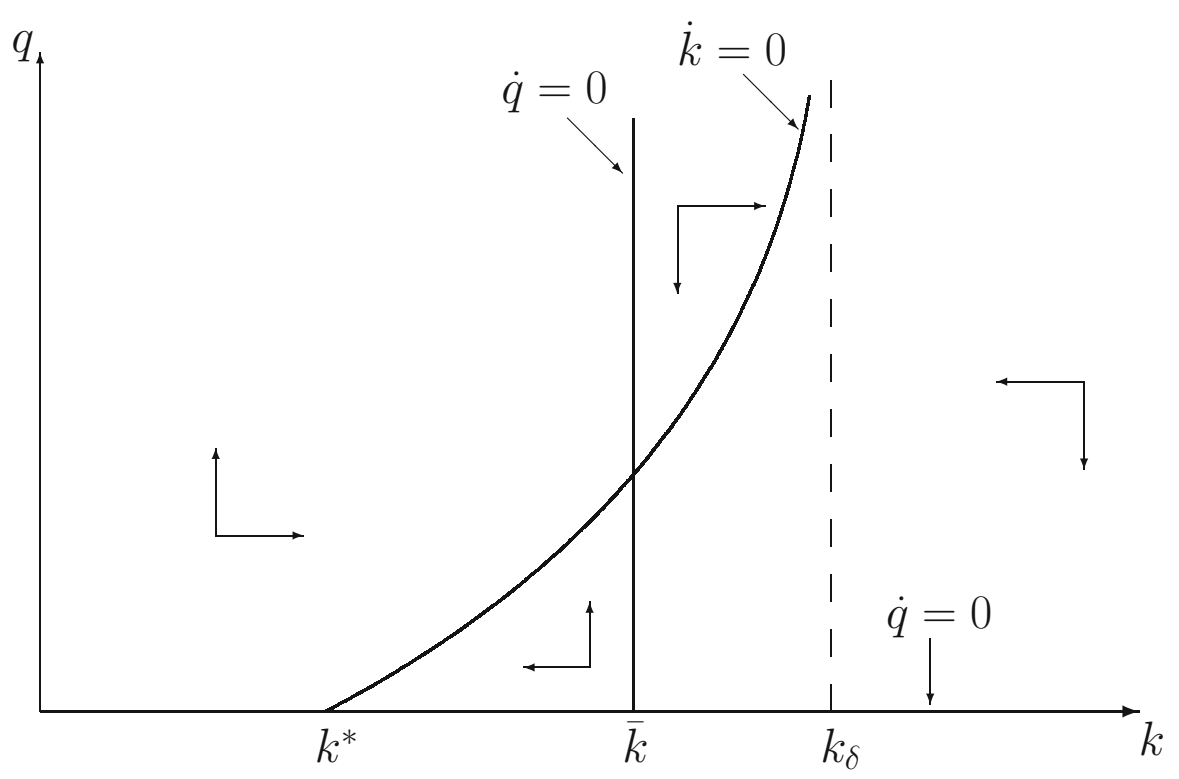

Fig. 9 The phase diagram for the case $n>0$ and $k^{*}<\bar{k}<k_{\delta}$ in Theorem 3(a)

After these preparatory remarks, we characterize the existence of bubbly equilibria in the following two theorems.

Theorem 3 Consider the extended Solow-Swan model consisting of (11) and (28)(29) and suppose that $n>0$ holds.

(a) If $k^{*}<\bar{k}$ is satisfied, then the standard Solow-Swan equilibrium is dynamically efficient. Nevertheless, there exist infinitely many dynamically efficient bubbly equilibria satisfying $\lim _{t \rightarrow+\infty}(k(t), q(t))=(\bar{k}, \bar{q})$.

(b) If $k^{*}>\bar{k}$ is satisfied, then the standard Solow-Swan equilibrium is dynamically inefficient. In addition there exist infinitely many dynamically inefficient bubbly equilibria satisfying $\lim _{t \rightarrow+\infty}(k(t), q(t))=\left(k^{*}, 0\right)$.

Proof First note that $n>0$ implies $\delta+n>\delta$ and, hence, $\bar{k}<k_{\delta}$.

(a) In this case it holds that $k^{*}<\bar{k}<k_{\delta}$. The isocline $\dot{k}(t)=0$ exists for all $k \in\{0\} \cup\left[k^{*}, K\right]$ if $k_{\delta}>K$ and for all $k \in\{0\} \cup\left[k^{*}, k_{\delta}\right)$ if $k_{\delta} \leq K$. It intersects the horizontal axis at the origin and at $k=k^{*}$, and it intersects the line $k=\bar{k}$ at $(\bar{k}, \bar{q})$. The phase diagram for this case is shown in Fig. 9. Because of $k^{*}<\bar{k}$, it follows that $\left(k^{*}, 0\right)$ is a saddle point and that its stable manifold coincides with the horizontal axis. Because $\bar{D}>0$ and $\bar{T}<0$ are satisfied, the fixed point $(\bar{k}, \bar{q})$ is a stable node or a stable focus. This implies that there exist infinitely many solutions of (11) and (28)-(29) which converge to $(\bar{k}, \bar{q})$. The statements about dynamic efficiency follow immediately from Proposition 1. Finally, one can see from Eqs. (28)-(29) that there do not exist equilibria with $\lim _{t \rightarrow+\infty} q(t)=+\infty$. Indeed, this could potentially only happen to the left of the vertical branch of the isocline $\dot{q}(t)=0$. If $q(t)$ were to grow 


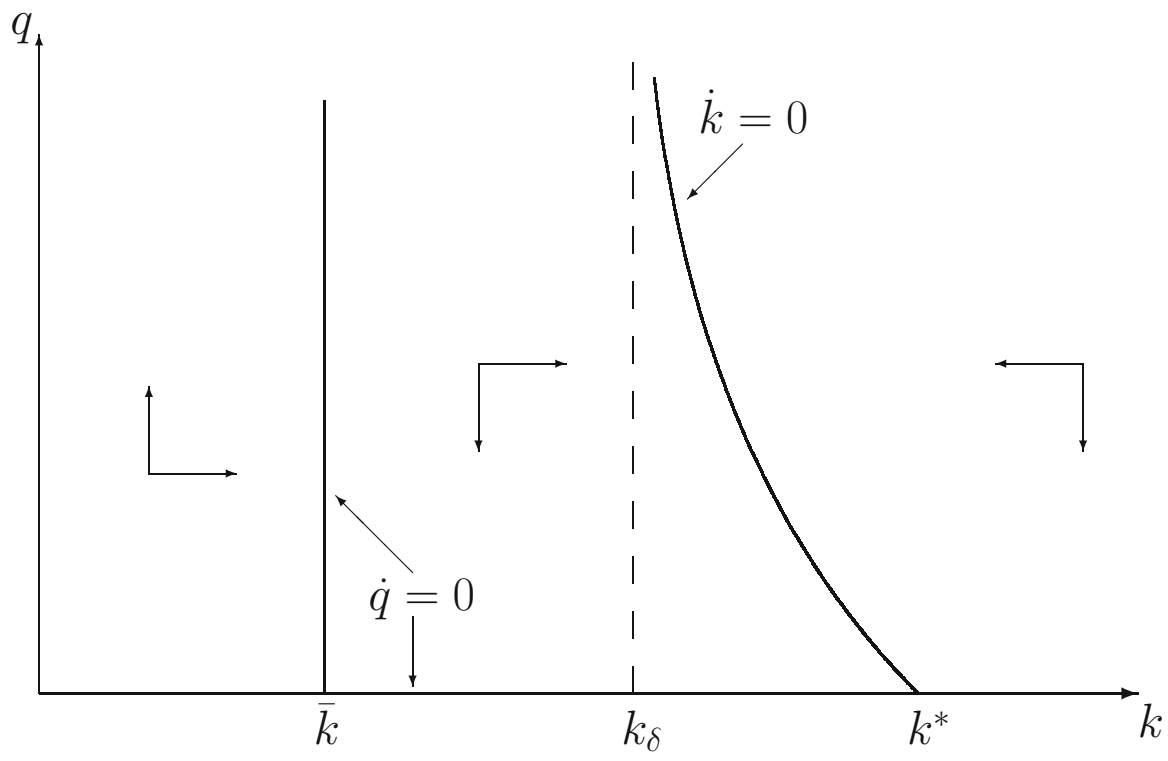

Fig. 10 The phase diagram for the case $n>0$ and $\bar{k}<k_{\delta}<k^{*}$ in Theorem 3(b)

without bound, the trajectory would leave $P_{+}$such that it would eventually hit the isocline $\dot{q}(t)=0$, from where on $q(t)$ would be decreasing.

(b) The case $\bar{k}<k^{*}$ consists of two subcases: $\bar{k}<k_{\delta}<k^{*}$ and $\bar{k}<k^{*}<k_{\delta}$. The phase diagrams are shown in Figs. 10 and 11, respectively. In both subcases, $\left(k^{*}, 0\right)$ is the only non-trivial fixed point and it is a stable node. This implies that there exist infinitely many solutions of (11) and (28)-(29) that converge to $\left(k^{*}, 0\right)$. All of them qualify as equilibria and, due to Proposition 1, all of them are dynamically inefficient. As in the proof of part (a) it can be shown that there are no other solutions of Eqs. (11) and (28)-(29).

As can be seen from the above theorem, in the present version of the model with an investment rule and a positive population growth rate there exist equilibria involving asset price bubbles independently of whether the standard Solow-Swan equilibrium is dynamically efficient or not.

Since the trace $\bar{T}$ of the Jacobian matrix $\bar{J}$ is always negative, it follows that Hopf bifurcations cannot occur in the present model. Thus, there is no easy way of demonstrating the existence of periodic solutions, if investment is determined by the rule (27). But damped oscillations are possible if $(\bar{k}, \bar{q})$ is a stable focus. This property, in turn, holds if and only if $\bar{D}>\bar{T}^{2} / 4$ is satisfied. To characterize the parameter region for which bubbly cycles exist in the present model, we define

$$
\begin{aligned}
& \phi_{1}=-\frac{n}{s F(\bar{k})-(\delta+n) \bar{k}}\left[(1-s)(\delta+n)-2 n-2 \sqrt{n^{2}-(1-s) n(\delta+n)}\right], \\
& \phi_{2}=-\frac{n}{s F(\bar{k})-(\delta+n) \bar{k}}\left[(1-s)(\delta+n)-2 n+2 \sqrt{n^{2}-(1-s) n(\delta+n)}\right] .
\end{aligned}
$$




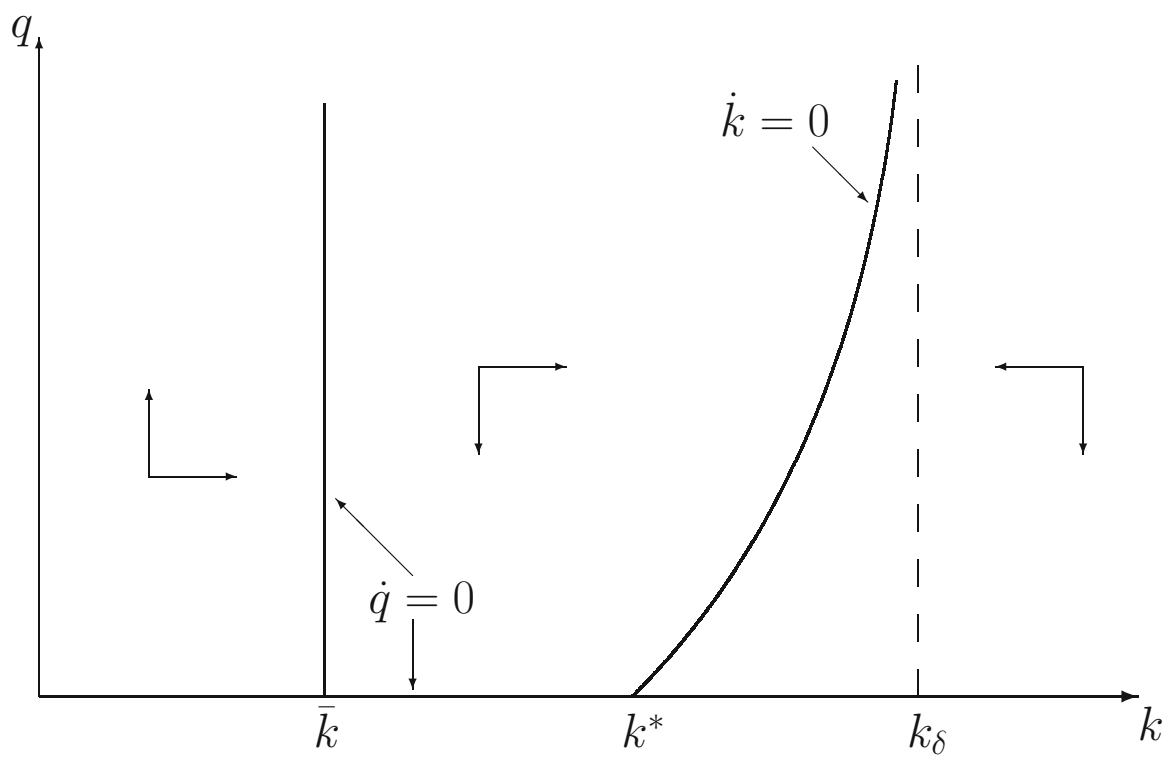

Fig. 11 The phase diagram for the case $n>0$ and $\bar{k}<k^{*}<k_{\delta}$ in Theorem 3(b)

Corollary 2 Suppose that $n>(1-s)(\delta+n)>0$ and $k^{*}<\bar{k}$ hold.

(a) If the inequality

$$
\phi_{1}<F^{\prime \prime}(\bar{k})<\phi_{2}
$$

is satisfied, then it follows that the bubbly equilibria described in theorem 3(a) display permanent but damped oscillations.

(b) If $F(k)=k^{\alpha}$ with $\alpha \in(0,1)$, then (36) cannot hold.

Proof (a) Using the expressions for $\bar{D}$ and $\bar{T}$ from (34) and (35), respectively, one can easily see that $\bar{D}=-n[\bar{T}+(1-s)(\delta+n)]$. The condition $\bar{D}>\bar{T}^{2} / 4$ is therefore equivalent to

$$
\bar{T}^{2}+4 n \bar{T}+4(1-s) n(\delta+n)<0
$$

This inequality holds if and only if $n>(1-s)(\delta+n)$ and

$$
-2 n-2 \sqrt{n^{2}-(1-s) n(\delta+n)}<\bar{T}<-2 n+2 \sqrt{n^{2}-(1-s) n(\delta+n)} .
$$

Using (35) and (31) it is straightforward to see that the latter inequality is equivalent to (36).

(b) If $F(k)=k^{\alpha}$, then it follows that the condition $k^{*}<\bar{k}$ is equivalent to $s<\alpha$. Furthermore, it is easy to see that Eq. (24) holds also in the present model, so that $\bar{D}-\bar{T}^{2} / 4>0$ is equivalent to 


$$
4(1-\alpha)\left(1-\frac{s}{\alpha}\right)-\left[s-1+\frac{(1-\alpha)(s-\alpha)(\delta+n)}{\alpha n}\right]^{2}>0 .
$$

The expression on the left-hand side of this inequality is a concave quadratic polynomial in $s$ and it is straightforward to verify that its maximum is negative provided that $n>0$.

The above corollary demonstrates that permanently oscillating bubbly equilibria are possible in the present model, but not if the production function is of Cobb-Douglas type. Let us now turn to the case of a negative population growth rate.

Theorem 4 Consider the extended Solow-Swan model consisting of (11) and (28)(29) and suppose that $-\delta<n<0$ holds.

(a) If $k^{*}<\bar{k}$ is satisfied, then the standard Solow-Swan equilibrium is dynamically efficient. In addition, there exist infinitely many dynamically efficient bubbly equilibria for which $\lim _{t \rightarrow+\infty}(k(t), q(t))=\left(k_{\delta},+\infty\right)$ holds.

(b) If $k^{*}>\bar{k}$ is satisfied, then the standard Solow-Swan equilibrium is dynamically inefficient and there exist equilibria involving asset price bubbles.

$\left(\mathrm{b}_{1}\right)$ There exists a unique bubbly equilibrium satisfying $\lim _{t \rightarrow+\infty}(k(t), q(t))=$ $(\bar{k}, \bar{q})$. This equilibrium is dynamically efficient.

$\left(\mathrm{b}_{2}\right)$ There exist infinitely many bubbly equilibria satisfying $\lim _{t \rightarrow+\infty}(k(t), q(t))=$ $\left(k^{*}, 0\right)$. These equilibria are dynamically inefficient.

$\left(\mathrm{b}_{3}\right)$ There exist infinitely many bubbly equilibria satisfying $\lim _{t \rightarrow+\infty}(k(t), q(t))=$ $\left(k_{\delta},+\infty\right)$. These bubbly equilibria are dynamically efficient.

Proof First note that $-\delta<n<0$ implies $\delta+n<\delta$ and, hence, $\bar{k}>k_{\delta}$.

(a) This case consists of the two subcases $k^{*}<k_{\delta}<\bar{k}$ and $k_{\delta}<k^{*}<\bar{k}$. The phase diagram for the subcase $k^{*}<k_{\delta}<\bar{k}$ is shown in Fig. 12. One can see that there exists only one non-trivial fixed point, namely $\left(k^{*}, 0\right)$. This fixed point is a saddle point, the stable manifold of which coincides with the horizontal axis. The stable saddle point path is the standard Solow-Swan equilibrium, which is dynamically efficient because of Proposition 1. In the present case, however, there exist solutions of (11) and (28)-(29), which do not approach a fixed point. Indeed, all trajectories that start from $q(0)>0$ will approach the unstable saddle point path of $\left(k^{*}, 0\right)$, which is upward sloping and located to the left of the isocline $\dot{k}(t)=0$. For such a solution it holds that $\lim _{t \rightarrow+\infty}(k(t), q(t))=\left(k_{\delta},+\infty\right)$, and it follows again from Proposition 1 that these equilibria are dynamically efficient.

In the second subcase it holds that $k_{\delta}<k^{*}<\bar{k}$. The corresponding phase diagram is shown in Fig. 13. This subcase is very similar to the previous one, except that the unstable saddle point path of $\left(k^{*}, 0\right)$ is downward sloping and located to the right of the isocline $\dot{k}(t)=0$.

(b) In this case it holds that $k_{\delta}<\bar{k}<k^{*}$. The phase diagram is shown in Fig. 14 and it is easily seen that the two isoclines have two non-trivial intersections, namely $\left(k^{*}, 0\right)$ and $(\bar{k}, \bar{q})$. The fixed point $\left(k^{*}, 0\right)$ is a stable node and the fixed point $(\bar{k}, \bar{q})$ is a saddle point. One can verify from the phase diagram using arguments analogous to those in the proof of part (a) that the solutions of (11) and (28)-(29) can be of the 


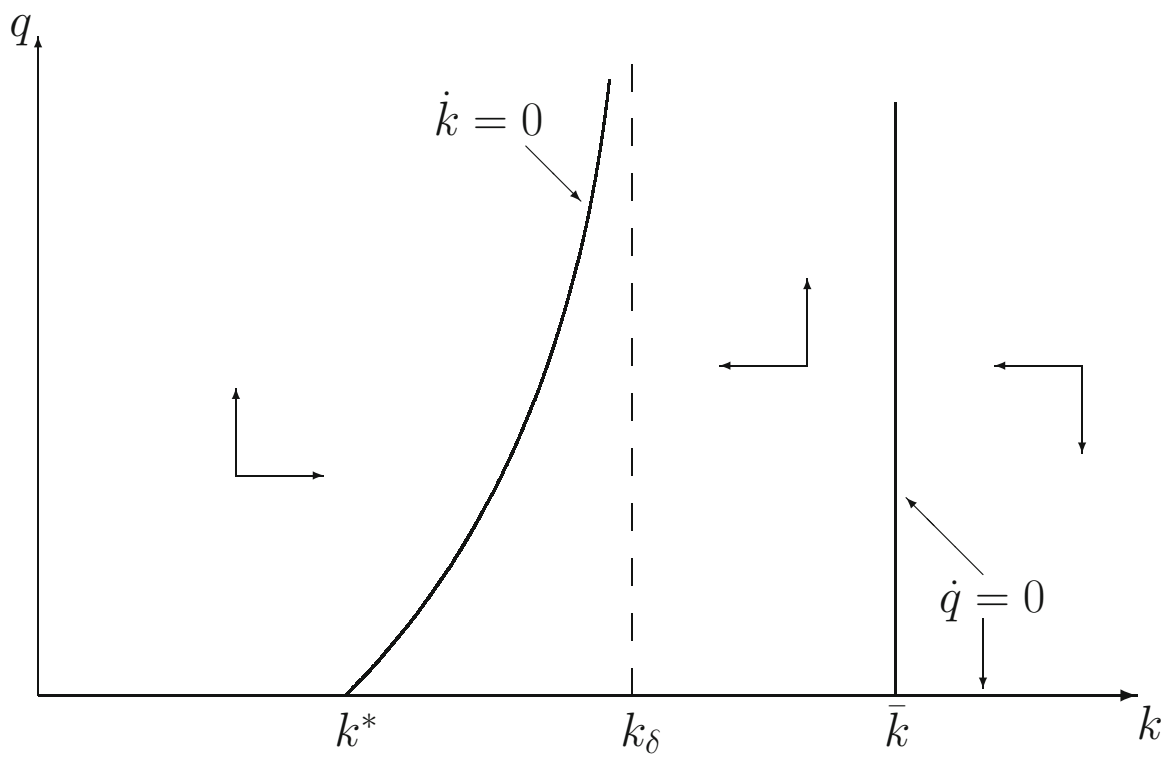

Fig. 12 The phase diagram for the case $-\delta<n<0$ and $k^{*}<k_{\delta}<\bar{k}$ in Theorem 4(a)

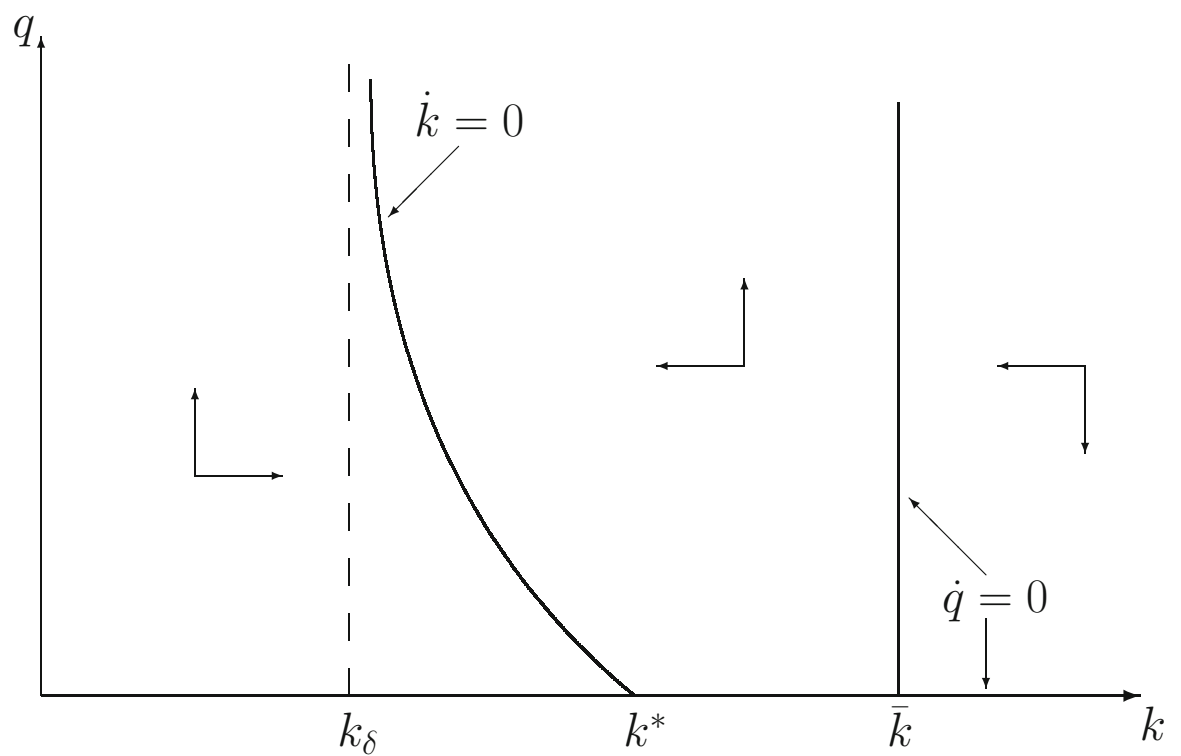

Fig. 13 The phase diagram for the case $-\delta<n<0$ and $k_{\delta}<k^{*}<\bar{k}$ in Theorem 4(a) 


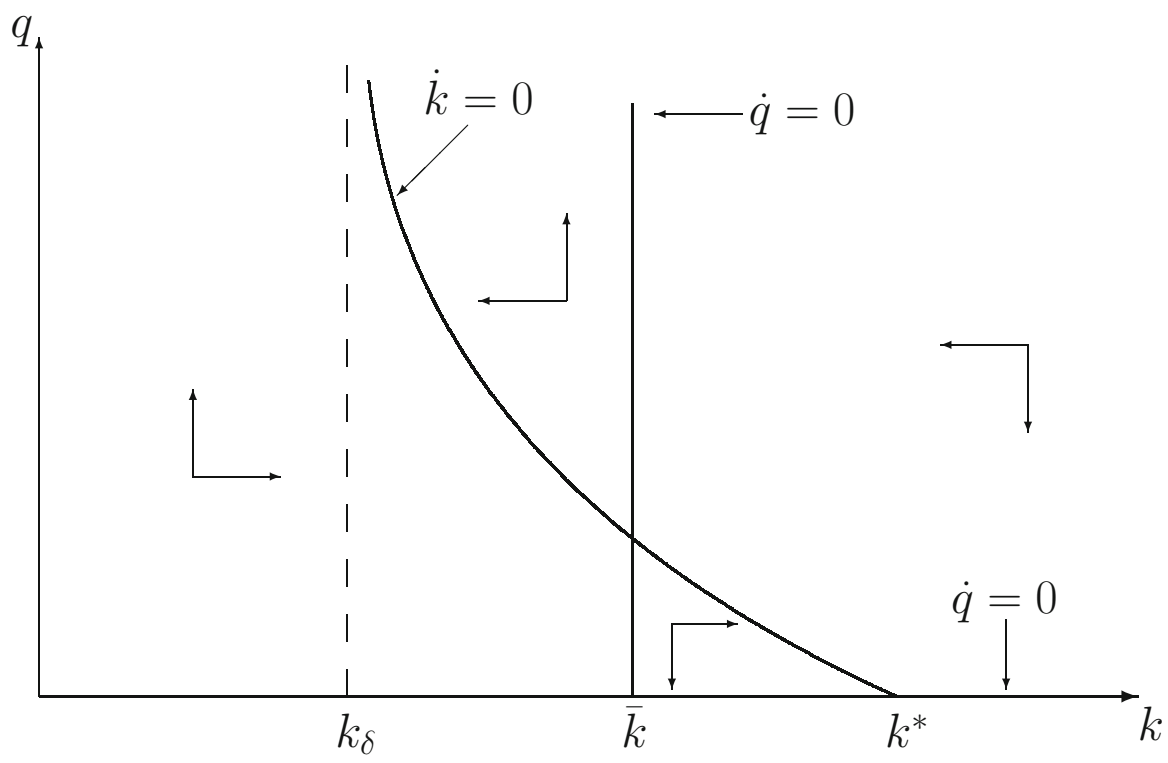

Fig. 14 The phase diagram for the case $-\delta<n<0$ and $k^{*}>\bar{k}$ in Theorem 4(b)

three types which are mentioned in items $\left(b_{1}\right),\left(b_{2}\right)$, and $\left(b_{3}\right)$. The dynamic efficiency properties follow from Proposition 1.

The above theorem shows that in the case $n<0$ there is a new type of bubbly equilibrium in which $q(t)$ grows without bound and $k(t)$ approaches $k_{\delta}$. Recalling equation (28) and the definition $q(t)=p(t) e^{-n t}$, it follows that the growth rate of $p(t)$, that is $\dot{p}(t) / p(t)$, approaches 0 as $t$ goes to infinity. As a matter of fact, along the line $k=k_{\delta}$ it holds that $\dot{p}(t)=0$.

\section{Concluding remarks}

This paper studies the existence and some properties of asset price bubbles in a neoclassical one-sector growth model with two assets: physical capital and an intrinsically useless asset. Following Solow (1956) and Swan (1956) we assume that consumption and investment are fixed fractions of total income, where the latter consists of GDP plus capital gains. We analyze under which conditions arbitrage-free pricing is consistent with a positive price of the useless asset. These conditions turn out to be different from those that ensure the dynamic inefficiency of the original Solow-Swan equilibrium. It is furthermore shown that asset price bubbles can result in non-monotonic, permanently oscillating, or even periodic paths of the per capita capital stock.

The Solow-Swan model lacks a micro foundation and the two behavioral rules considered in the present paper (a consumption rule and an investment rule) can be criticized as being ad hoc. Nevertheless, we hope that the results of our analysis shed some light on the behavioral patterns that favor the emergence of asset price bubbles. In 
particular, the possibility that capital gains directly affect the demand for consumption goods or investment goods seems to be intuitively plausible. Our analysis has shown that such a feedback mechanism may be responsible for asset price bubbles. In future research we will try to find variants of other standard neoclassical growth models in which the direct feedback of capital gains on investment demand can be microfounded. The most promising framework for this endeavor would be a model with overlapping generations but, as has been pointed out in the introduction, one would have to consider a version in which households make a consumption/saving decision at a time when they already have asset income. This rules out models with two-period lived households as in Diamond (1965) or Tirole (1985).

Another possible direction for future research would be to consider a broad class of behavioral rules (say, all rules of the Markovian form $c(t)=h(k(t), q(t)))$ and to characterize those properties of these rules that are consistent with the existence of asset price bubbles. Finally, it would be interesting to analyze how the possibility that a price bubble can burst affects the dynamics. We believe that this is likely to generate very rich dynamics which can hopefully still be studied by the graphical methods applied in the present paper.

Acknowledgements Open access funding provided by University of Vienna.

Open Access This article is distributed under the terms of the Creative Commons Attribution 4.0 International License (http://creativecommons.org/licenses/by/4.0/), which permits unrestricted use, distribution, and reproduction in any medium, provided you give appropriate credit to the original author(s) and the source, provide a link to the Creative Commons license, and indicate if changes were made.

\section{References}

Acemoglu D (2009) Introduction to modern economic growth. Princeton University Press, Princeton

Aliber RZ, Kindleberger CP (2015) Manias, panics, and crashes: a history of financial crises, 7th edn. Palgrave Macmillan, Basingstoke

Blanchard OJ (1985) Debt, deficits, and finite horizons. J Polit Econ 93:223-247

Caballero RJ, Farhi E, Hammour ML (2006) Speculative growth: hints from the U.S. economy. Am Econ Rev 96:1159-1192

Cass D (1972) On capital overaccumulation in the aggregative, neoclassical model of economic growth: a complete characterization. J Econ Theory 4:200-223

Diamond P (1965) National debt in a neoclassical growth model. Am Econ Rev 55:1126-1150

Farhi E, Tirole J (2012) Bubbly liquidity. Rev Econ Stud 79:678-706

Guckenheimer J, Holmes P (1983) Nonlinear oscillations, dynamical systems, and bifurcations of vector fields. Springer, Berlin

Kocherlakota NR (1992) Bubbles and constraints on debt accumulation. J Econ Theory 57:245-256

Kocherlakota NR (2009) Bursting bubbles: consequences and cures. Paper prsented at the IMF conference macroeconomic and policy challenges following financial meltdowns, April 3

Martin A, Ventura J (2012) Economic growth with bubbles. Am Econ Rev 102:3033-3058

Martin A, Ventura J (2016) Managing credit bubbles. J Eur Econ Assoc 14:753-789

Miao J (2014) Introduction to economic theory of bubbles. J Math Econ 53:130-136

Samuelson PA (1958) An exact consumption-loan model of interest with or without the social contrivance of money. J Polit Econ 66:467-482

Scherbina A, Schlusche B (2014) Asset price bubbles: a survey. Quant Finance 14:589-604

Solow RM (1956) A contribution to the theory of economic growth. Q J Econ 70:65-94

Swan TW (1956) Economic growth and capital accumulation. Econ Rec 32:334-361

Tirole J (1985) Asset bubbles and overlapping generations. Econometrica 53:1071-1100 
Weil P (1987) Confidence and the real value of money in an overlapping generations economy. Q J Econ 102:1-22

Yaari M (1965) Uncertain lifetime, life insurance, and the theory of the consumer. Rev Econ Stud 32:137150 